\title{
The accretion environment of supergiant fast X-ray transients probed with XMM-Newton
}

\author{
E. Bozzo ${ }^{1}$, F. Bernardini ${ }^{2}$, C. Ferrigno ${ }^{1}$, M. Falanga ${ }^{3}$, P. Romano ${ }^{4}$, and L. Oskinova ${ }^{5,6}$ \\ 1 Department of Astronomy, University of Geneva, Chemin d'Ecogia 16, 1290 Versoix, Switzerland \\ e-mail: enrico.bozzo@unige.ch \\ 2 New York University Abu Dhabi, PO Box 129188 Abu Dhabi, UAE \\ 3 International Space Science Institute (ISSI) Hallerstrasse 6, 3012 Bern, Switzerland \\ 4 INAF-Osservatorio Astronomico di Brera, via Emilio Bianchi 46, 23807 Merate (LC), Italy \\ 5 Institut für Physik und Astronomie, Universität Potsdam, Karl-Liebknecht-Strasse 24/25, 14476 Potsdam, Germany \\ 6 Kazan Federal University, Kremlevskaya Str., 18, 420008 Kazan, Russia
}

Received 5 January 2017 / Accepted 31 August 2017

\begin{abstract}
Context. Supergiant fast X-ray (SFXT) transients are a peculiar class of supergiant X-ray binaries characterized by a remarkable variability in the X-ray domain, widely ascribed to accretion from a clumpy stellar wind.

Aims. In this paper we performed a systematic and homogeneous analysis of the sufficiently bright X-ray flares observed with $X M M-N e w t o n$ from the supergiant fast X-ray transients to probe spectral variations on timescales as short as a few hundred seconds. Our ultimate goal is to investigate whether SFXT flares and outbursts are triggered by the presence of clumps, and to reveal whether strongly or mildly dense clumps are required.

Methods. For all sources, we employ a technique developed by our group already exploited in a number of our previous papers, making use of an adaptive rebinned hardness ratio to optimally select the time intervals for the spectral extraction. A total of twelve observations performed in the direction of five SFXTs are reported, providing the largest sample of events available so far.

Results. Using the original results reported here and those obtained with our technique from the analysis of two previously published XMM-Newton observations of IGR J17544-2619 and IGR J18410-0535, we show that both strongly and mildly dense clumps can trigger these events. In the former case, the local absorption column density may increase by a factor of $\gg 3$, while in the latter case, the increase is only a factor of $\sim 2-3$ (or lower). An increase in the absorption column density is generally recorded during the rise of the flares/outbursts, while a drop follows when the source achieves peak flux. In a few cases, a re-increase of the absorption column density after the flare is also detected, and we discovered one absorption event related to the passage of an unaccreted clump in front of the compact object. Overall, there seems to be no obvious correlation between the dynamic ranges in the X-ray fluxes and absorption column densities in supergiant fast X-ray transients, with an indication that lower densities are recorded at the highest fluxes.

Conclusions. The spectral variations measured in all sources are in agreement with the idea that the flares/outbursts are triggered by the presence of dense structures in the wind interacting with the X-rays from the compact object (leading to photoionization). The lack of correlation between the dynamic ranges in the X-ray fluxes and absorption column densities can be explained by the presence of accretion inhibition mechanism(s). Based on the knowledge acquired so far on the SFXTs, we propose a classification of the flares/outbursts from these sources in order to drive future observational investigations. We suggest that the difference between the classes of flares/outbursts is related to the fact that the mechanism(s) inhibiting accretion can be overcome more easily in some sources compared to others. We also investigate the possibility that different stellar wind structures, other than clumps, could provide the means to temporarily overcome the inhibition of accretion in supergiant fast X-ray transients.
\end{abstract}

Key words. X-rays: individuals: IGRJ18450-0435 - X-rays: individuals: IGRJ17544-2619 - X-rays: binaries -

X-rays: individuals: SAXJ1818.6-1703 - X-rays: individuals: IGRJ17354-3255 - X-rays: individuals: IGRJ16328-4726

\section{Introduction}

Supergiant fast X-ray transients (SFXTs) are a subclass of the classical supergiant high mass X-ray binaries ( $\mathrm{SgXBs}$ ) hosting a neutron star (NS) accreting from the wind of an O-B supergiant companion (see, e.g., Martínez-Núñez et al. 2017, for a recent review). At odds with classical systems, the SFXTs are known to be largely subluminous (a factor of $\gtrsim 10-100$ ) and to display a much more prominent variability in X-rays. In particular, SFXTs spend most of their lifetimes in low quiescent states (with X-ray luminosities as low as $10^{32} \mathrm{erg} \mathrm{s}^{-1}$ ) and undergo sporadic outbursts of a few hours each reaching luminosities of $L_{\mathrm{X}} \simeq 10^{36}-10^{38} \mathrm{erg} \mathrm{s}^{-1}$. Flares with intermediate luminosities between quiescence and outburst $\left(L_{\mathrm{X}} \simeq 10^{34}-10^{35} \mathrm{erg} \mathrm{s}^{-1}\right)$ are often observed and last a few thousands seconds at most (see, e.g., Romano et al. 2014). The largest dynamic range in luminosity recorded so far is that of the SFXT prototype IGR J175442619 , reaching a factor of $\sim 10^{6}$ (Romano et al. 2015). For comparison, the classical $\mathrm{SgXBs}$ display a typical dynamic range of $\lesssim 100$ (Walter et al. 2015).

It was originally proposed that largely eccentric orbits and extremely clumpy winds could explain the SFXT phenomenology (in 't Zand 2005; Negueruela 2010; Walter \& Zurita Heras 2007). In a wide elongated orbit, the NS remains quiescent for a significant fraction of time, and the accretion of clumps endowed with an overdensity of a factor of $\gtrsim 1000$ compared to the surrounding wind could give rise to the brightest SFXT 
outbursts (especially when the NS approaches the companion). The problem with this interpretation is that a number of SFXTs were discovered to have short orbital periods (35 days), and studies of isolated supergiant winds showed that clumps are typically only a factor of $\simeq 10$ overdense compared to a smooth wind (see, e.g., Lutovinov et al. 2013; Bozzo et al. 2015; Martínez-Núñez et al. 2017, and references therein). Bozzo et al. (2008b) and Grebenev \& Sunyaev (2007) proposed that the inhibition of accretion caused by the neutron star rotating magnetosphere could combine with the presence of moderately dense clumps to boost the X-ray variability of the SFXTs compared to classical systems. An alternative way to inhibit accretion and widen the dynamic range of the SFXTs involve the combined presence of a long-lasting "subsonic settling accretion regime" and a significantly magnetized supergiant wind (Shakura et al. 2012, 2014).

Although the theoretical interpretation of the SFXT behavior is still matter of debate, convincing evidence has been reported in the literature of the presence of massive structures in the stellar winds around the NSs in these systems. Observationally, the role of these structures is twofold. A sufficiently large and dense structure simply passing in front of the NS (without being accreted) causes source dimming or even obscuration. Its presence can be revealed by the signature of the photoelectric absorption, even when there is no significant increase in the source X-ray emission. Structures that instead are intercepted by the NS lead to a temporarily higher mass accretion rate, giving rise to X-ray flares or outbursts characterized by an enhanced local absorption that is proportional to the lateral size of the accreted structure (see, e.g., the discussion in Bozzo et al. 2013). Events of source dimming have been reported in several observations of SFXTs and tentatively associated with clumps passing in front of the compact object (see, e.g., Rampy et al. 2009; Drave et al. 2013). X-ray flares and outbursts in SFXTs have also been associated with accretion of clumps in several publications, but finding direct proof of the existence of these structures through X-ray observations has proved challenging. The reason is that SFXT flares and outbursts occur at unpredictable times. Their detailed spectroscopic analysis requires monitoring with a large effective area instrument able to give access to integrations as short as the local dynamical timescales (hundreds of seconds) with the necessary signal-to-noise ratio $(\mathrm{S} / \mathrm{N})$ and energy resolution.

So far, the most convincing evidence for the "ingestion" of a massive clump by a NS hosted in a SFXT was reported by Bozzo et al. (2011) using an XMM-Newton observation that luckily caught IGR J18410-0535 undergoing a bright X-ray flare. On that occasion, a a factor of $\sim 10$ increase in the $a b$ sorption column density in the direction of the source preceding the flare revealed that a massive and dense structure was rapidly approaching the NS, giving first rise to a large increase in flux (a factor of 1000) before obscuring the compact object and finally moving away from the observer's line of sight. Bozzo et al. (2016a) also fortuitously caught with XMM-Newton an episode of strongly enhanced X-ray activity from the SFXT IGR J175442619 that reached the typical luminosity of an X-ray outburst, but displayed only a marginal increase in the local absorption column density (a factor of $\lesssim 2$ ). Relibly estimating the physical properties of the clumps from these observations have proved complicated, due to our poor knowledge of the spin period and magnetic field of the NS in the SFXTs. This prevents us from correctly quantifying the effect of the mechanisms inhibiting accretion and contributing to producing the total variation of the
X-ray luminosity (see also the detailed discussion in Bozzo et al. 2011).

In this paper, we analyze all publicly available XMM-Newton observations of confirmed SFXTs in which sufficiently bright X-ray flares/outbursts are detected to perform a detailed spectral analysis. We take advantage of the large collecting area of the EPIC cameras on board XMM-Newton (Jansen et al. 2001; Strüder et al. 2001; Turner et al. 2001) to investigate spectral variations during flares that can be associated with the presence of structures in the wind of the supergiant companion. An adaptively rebinned hardness-ratio resolved spectral analysis is homogeneously carried out for a total of 12 observations performed in the direction of 5 out of the $\sim 10$ known SFXTs (IGR J184500435, IGR J17544-2619, SAX J1818.6-1703, IGR J17354-3255, and IGR J16328-4726). Our dataset includes data that were published previously but not investigated for rapid spectral variations and also five unpublished observations. The unique capability of XMM-Newton for these studies is to provide a sufficiently large effective area in the soft X-ray domain $(0.5-12 \mathrm{keV})$ to perform spectroscopic investigations down to timescales as short as a few hundred seconds, thus allowing us to resolve changes in the photoelectric absorption in the direction of the source during the rise and decays of the flares (and in the quiescent periods between them). Based on our experience, we consider flares to be sufficiently bright when the source count-rate achieves at least $2-3$ cts s $^{-1}$ in the EPIC cameras ${ }^{1}$.

Our final goal is to test whether all observed flares/outbursts are characterized by a spectral variability that can be associated with the presence of a massive clump around the compact object and whether less massive structures are also able to trigger these phenomena. We summarize in Sect. 2 the data analysis procedure and describe in the associated subsections the results obtained for each source and each observation. An overview of the results is provided in Sect. 3. The corresponding discussion is presented in Sect. 4, together with our conclusions.

\section{XMM-Newton data reduction and analysis}

For all sources considered in this paper, we produced calibrated event lists by using the Science Analysis System (SAS) v.15.0. The EPIC-pn, EPIC-MOS, and RGS event files were generated with standard analysis performed with the EPPROC, EMPROC, and RGSPROC tasks. The EPIC-pn and EPIC-MOS data were filtered in the $0.5-12 \mathrm{keV}$ and $0.5-10 \mathrm{keV}$ energy range, respectively. Extraction regions for the light curves and spectra of all sources and background were selected by following recommendations in the latest SAS analysis threads ${ }^{2}$. The difference in extraction areas between source and background was accounted for by using the SAS BACKSCALE task for the spectra and LCCORRR for the light curves. The EPIC spectra were rebinned before fitting in order to have 15-25 counts per bin (depending on the available statistics) and, at the same time, to prevent oversampling of the energy resolution by more than a factor of three. The RGS spectra were also rebinned accordingly, whenever required. The timing analysis of all EPIC data was performed on barycenter-corrected event lists (we used the SAS barycen tool). Details of specific issues regarding the observations of the different sources are provided in the dedicated following sections.

\footnotetext{
1 The only exception in this paper is observation 0679810401, where we were able to show a modest (but still significant) variation in the absorption column density during a faint X-ray flare from IGR J175442619.

2 http://xmm. esac. esa.int/sas/current/documentation/
} threads 
Throughout this paper, uncertainties are given at $90 \%$ confidence level, unless otherwise stated.

For all sources, we searched for rapid spectral changes in their X-ray emission by performing a hardness ratio (HR) resolved spectral analysis. The HR is computed in all cases from the adaptively rebinned energy-resolved light curves and calculated as the ratio of the source count-rate in the soft to hard energy bands vs. time. The adaptive rebinning and the choice of the soft and hard energy bands are performed differently for each source, depending on the data statistics and the averaged spectral properties. The HR-resolved spectral analysis we employ in this paper is better suited to revealing rapid spectral variations during the flares/outbursts of the SFXTs than the more standard intensity or time-resolved spectral analyses carried out previously for 7 (out of 12) X-ray observations included in our study. The HR-resolved spectral analysis probes directly when a source is undergoing a significant spectral variation, without the need to assume a priori that such changes can occur either during periods of lower/higher X-ray activity or in specific time intervals. This technique was already exploited in two of our previous publications and allowed us to prove that the bright flare observed by XMM-Newton from the SFXT IGR J18410-0535 was most likely related to the ingestion of a massive clump by the NS hosted in this system (Bozzo et al. 2011), while the outburst caught from the SFXT prototype IGR J17544-2619 was shown to require the simultaneous interplay of additional mechanisms to give rise to the measured dynamic range in the X-ray luminosity (Bozzo et al. 2016a). The conclusion in the case of IGR J175442619 was mainly derived from the fact that no particularly large increase in the accretion column density was recorded during this event, although it achieved an X-ray luminosity a factor of $\gtrsim 10$ higher than that of the X-ray flare caught from IGR J184100535 which displayed evidence of an increase in the local $N_{\mathrm{H}}$ of a factor of $\sim 10$ preceding the $\mathrm{X}$-ray flare. We note that for all sources analyzed in this paper the estimated distance is of $\sim 2-$ 4 kpc (Martínez-Núñez et al. 2017, and references therein), and thus all episodes of enhanced X-ray emission reported in the next sections are compatible with being flares as the peak luminosity is in all cases $\lesssim 10^{36} \mathrm{erg} \mathrm{s}^{-1}$.

The soft X-ray spectra of almost all sources considered here are well described by using an absorbed power-law model, as is commonly the case for SFXTs and other HMXBs. The cutoff of the power law at high energies is usually measurable only when a broadband spectrum extending up to $\gtrsim 20-30 \mathrm{keV}$ is available (see, e.g., Walter et al. 2015, and references therein). The 1-10 keV X-ray flux was measured by using the PEGPOW model in XSPEC in order to have values not dependent on the largely variable absorption column densities and the uncertainties that arise when the spectra are de-absorbed after the fit in XSPEC.

Supergiant fast X-ray transients are characterized (on average) by a relatively high absorption column density $\left(\gg 10^{22} \mathrm{~cm}^{-2}\right)$, well in excess of the galactic extinction in the source direction. However, the generally low statistics of the data at energies below 2-3 keV do not allow us to unambiguously separate the contribution of the Galactic absorption and the additional absorption local to the source. The former contribution can be roughly estimated by using an online tool ${ }^{3}$ based on the results published by Dickey \& Lockman (1990) or by more recent works that also consider the contribution of molecular hydrogen (Dame et al. 2001). The density of absorbing material located close to the source, which is generally largely in excess

\footnotetext{
3 http://heasarc.gsfc . nasa.gov/cgi-bin/Tools/w3nh/ w3nh.pl?
}

of the expected Galactic value, can be obtained by measuring an excess with respect to the expected Galactic value using a simple PHABS spectral component in XSPEC. This is a common practice in the case of HMXBs for which high statistics X-ray observations with grating spectrometers are not available (see, e.g., Grinberg et al. 2015). In a few observations used in this work, slightly more complex spectral models were also considered, including a partial covering absorber (PCFABS in XSPEC). This model is also widely used to describe the soft X-ray emission of HMXBs, and it is usually ascribed to localized absorbers that partially intercept the radiation from the NS (see, e.g., Tomsick et al. 2009, and references therein). The X-ray emission that escapes this extra absorption is only affected by the Galactic column density. The fits to the spectra of a few sources also required the addition of a thin Gaussian line with a centroid energy compatible with that expected from neutral iron at $6.4 \mathrm{keV}$. Similar lines in HMXBs are known to occur and are due to the fluorescence of the X-ray photons released by the NS onto the surrounding stellar wind material (see, e.g., Torrejón et al. 2010; Giménez-García et al. 2016). We refer the reader to the following subsections dedicated to each source for further details.

The log of all XMM-Newton observations used in this paper is given in Table 1. For completeness, we also add in Table 2 the XMM-Newton observations that we published previously for which an adaptively rebinned HR-resolved spectral analysis of the SFXT flares/outbursts was already carried out. These observations are used in Sect. 4 to provide a more exhaustive discussion and conclusions of all results.

\subsection{IGRJ18450-0435}

\subsubsection{Observation 0306170401}

This observation was first analyzed by Zurita Heras \& Walter (2009). It was not affected by high flaring background interval and thus the entire exposure time can be retained for the scientific analysis. By using the EPATPLOT tool, we found that the source X-ray emission was moderately affected by pileup at count-rates $\gtrsim 7$ cts s $^{-1}$ on both the EPIC-pn and the MOS cameras. We verified that using an annular extraction region with a inner radius of $100 \mathrm{px}$ for the EPIC-pn and $150 \mathrm{px}$ for the MOS could remove the effect of pileup. Figure 1 (top) shows the source energy-resolved light curves, together with the corresponding HR. This is also shown in the same figure as a function of the total source intensity (hardness intensity diagram, HID). No apparent correlation emerges between the source count-rate and the HR, but some specific intervals of count-rates are visible where the variations of the HR are more pronounced. We extracted the pileup corrected EPIC spectra and also made use of the RGS1 and RGS2 average spectra summed with the SAS tool RGSCOMBINE to improve the S/N.

In order to carry out a comparison with previously reported results, we also extracted the spectrum from the IBIS/ISGRI instrument on board INTEGRAL during the seven pointings considered by Zurita Heras \& Walter (2009, see their Table 2). The IBIS/ISGRI data were analyzed by using standard procedures and the version 10.2 of the OSA software distributed by the ISDC (Courvoisier et al. 2003).

In Fig. 2 we show the averaged EPIC-pn and MOS spectra summed together by following the SAS online threads ${ }^{4}$ in order to achieve the highest possible $\mathrm{S} / \mathrm{N}$. This spectrum was

\footnotetext{
4 http://xmm. esac. esa. int/sas/current/documentation/ threads/
} 
Table 1. Log of all XMM-Newton observations considered in this paper for the different SFXT sources.

\begin{tabular}{|c|c|c|c|c|c|c|}
\hline Source & Obs. ID & $\begin{array}{l}\text { Start } \\
(\mathrm{UTC})\end{array}$ & $\begin{array}{l}\text { Stop } \\
\text { (UTC) }\end{array}$ & $\begin{array}{l}\text { Exp. } \\
(\mathrm{ks})\end{array}$ & Reference & Comments \\
\hline IGR J18450-0435 & $\begin{array}{l}0306170401 \\
0728370801 \\
0728371001\end{array}$ & $\begin{array}{l}2006-04-03 \quad 14: 44 \\
2014-10-13 \quad 23: 29 \\
2014-10-15 \quad 16: 20\end{array}$ & $\begin{array}{l}2006-04-03 \text { 20:22 } \\
2014-10-1405: 50 \\
2014-10-15 \text { 21:50 }\end{array}$ & $\begin{array}{l}19.0 \\
21.6 \\
18.5\end{array}$ & $\begin{array}{l}\text { Zurita Heras \& Walter (2009) } \\
\text { this work } \\
\text { this work }\end{array}$ & $\begin{array}{l}\text { bright flares } \\
\text { bright flares } \\
\text { faint flares }\end{array}$ \\
\hline IGR J17544-2619 & $\begin{array}{l}0148090501 \\
0154750601 \\
0679810401\end{array}$ & $\begin{array}{l}2003-09-11 \quad 18: 44 \\
2003-09-1717: 13 \\
2012-09-1602: 55\end{array}$ & $\begin{array}{l}2003-09-1122: 09 \\
2003-09-11 \text { 19:59 } \\
2012-09-1607: 30\end{array}$ & $\begin{array}{l}11.0 \\
8.0 \\
15.0\end{array}$ & $\begin{array}{l}\text { González-Riestra et al. (2004) } \\
\text { González-Riestra et al. (2004) } \\
\text { Drave et al. (2014) }\end{array}$ & $\begin{array}{l}\text { bright flares } \\
\text { bright flares, affected by high background } \\
\text { rise to a flaring state }\end{array}$ \\
\hline SAX J1818.6-1703 & 0693900101 & 2013-03-21 12:19 & 2013-03-21 21:56 & 33.3 & Boon et al. (2016) & $\begin{array}{l}\text { bright flares, moderately affected by high back- } \\
\text { ground }\end{array}$ \\
\hline IGR J17354-3255 & $\begin{array}{l}0693900201 \\
0701230101 \\
0701230701\end{array}$ & $\begin{array}{l}2013-03-1512: 47 \\
2013-03-2911: 31 \\
2013-03-3102: 17\end{array}$ & $\begin{array}{l}2013-03-1522: 22 \\
2013-03-2921: 22 \\
2013-03-3108: 41\end{array}$ & $\begin{array}{l}33.2 \\
34.2 \\
21.7\end{array}$ & $\begin{array}{l}\text { this work } \\
\text { this work } \\
\text { this work }\end{array}$ & $\begin{array}{l}\text { flares, moderately affected by high background } \\
\text { flares, moderately affected by high background } \\
\text { several flares }\end{array}$ \\
\hline IGR J16328-4726 & $\begin{array}{l}0728560201 \\
0728560301\end{array}$ & $\begin{array}{l}\text { 2014-08-24 19:17 } \\
\text { 2014-08-26 18:44 }\end{array}$ & $\begin{array}{l}2014-08-2505: 20 \\
2014-08-2701: 07\end{array}$ & $\begin{array}{l}34.9 \\
21.7\end{array}$ & $\begin{array}{l}\text { Fiocchi et al. (2016) } \\
\text { Fiocchi et al. (2016) }\end{array}$ & $\begin{array}{l}\text { moderately bright flares } \\
\text { moderately bright flares }\end{array}$ \\
\hline
\end{tabular}

Notes. We provide the relevant reference for the observations already published by other authors (without performing an adaptively rebinned HRresolved spectral analysis) and marked with "this work" data that are publicly available but were not published yet. In the column "Comments" we indicated if the source was showing bright or moderately bright flares, providing also some information about the level of background in the most critical cases.

Table 2. Log of all XMM-Newton observations of flaring/outbursting SFXTs published previously and for which an adaptively rebinned HRresolved spectral analysis was already carried out.

\begin{tabular}{lllllll}
\hline \hline Source & Obs. ID & $\begin{array}{l}\text { Start } \\
(\text { UTC) }\end{array}$ & $\begin{array}{l}\text { Stop } \\
(\text { UTC) }\end{array}$ & $\begin{array}{l}\text { Exp. } \\
(\mathrm{ks})\end{array}$ & Reference & Comments \\
\hline IGR J17544-2619 & 0744600101 & $2015-03-2005: 00$ & $2015-03-21$ 20:17 & 140.0 & Bozzo et al. (2016a) & outburst \\
\hline IGR J18410-0535 & 0604820301 & $2010-03-15 ~ 13: 09$ & $2010-03-1602: 13$ & 45.7 & Bozzo et al. (2011) & bright flare \\
\hline
\end{tabular}

grouped in order to have at least 150 photons per energy bin. We verified a posteriori that results obtained from the fit to this spectrum were compatible with those obtained by using only the EPIC-pn spectrum. A fit to the summed EPIC spectrum was carried out simultaneously with the RGS and the IBIS/ISGRI spectra, first by using the same spectral model considered by Zurita Heras \& Walter (2009). This model comprises an emission from optically thin plasma at the lower energies (MEKAL in XSPEC), a cutoff power law to fit the data above $\sim 4 \mathrm{keV}$, and an edge at $\sim 7.9 \mathrm{keV}$. We added to this model a Gaussian line at $\sim 6.4 \mathrm{keV}$, which had previously gone unnoticed, and the intercalibration constants for IBIS/ISGRI and the RGS with respect to the summed EPIC cameras. These constants also account for the source variability as the IBIS/ISGRI and XMM-Newton data are not strictly simultaneous. This model gave a reasonable description of the data and parameter values in agreement with those previously reported by Zurita Heras \& Walter (2009). We found that a simpler model comprising a partial covering absorber, a cutoff power law, and an iron line could describe the data equally well $\left(\chi_{\text {red }}^{2} /\right.$ d.o.f. $\left.=1.14 / 325\right)$. We measured in this case $N_{\mathrm{H}}^{\mathrm{los}}=(1.8 \pm 0.1) \times 10^{22} \mathrm{~cm}^{-2}, N_{\mathrm{H}}=\left(5.5_{-1.0}^{+1.2}\right) \times 10^{22} \mathrm{~cm}^{-2}$, $f=5.6 \pm 0.4, \Gamma=1.3 \pm 0.1, E_{\text {cut }} 31.7_{-9.2}^{+16.9} \mathrm{keV}, E_{\mathrm{Fe}}=$ $6.42 \pm 0.02 \mathrm{keV}, E Q W_{\mathrm{Fe}}=0.05_{-0.01}^{+0.02}, C_{\mathrm{RGS}}=1.1 \pm 0.1$, and $C_{\text {IBIS/ISGRI }}=4.8 \pm 1.0$. Here $N_{\mathrm{H}}^{\mathrm{los}}$ is the Galactic column density, $N_{\mathrm{H}}$ the column density of the partial covering absorber, $f$ the covering fraction, $E_{\text {cut }}$ the cutoff energy of the cutoff powerlaw component, $\Gamma$ the corresponding photon index, $E_{\mathrm{Fe}}$ is the centroid energy of the iron line, and $E Q W_{\mathrm{Fe}}$ is its equivalent width. We fixed the normalization constant of the combined
EPIC cameras spectrum to unity and indicated with $C_{\mathrm{RGS}}$ and $C_{\text {IBIS/ISGRI }}$ the values of the normalization constants obtained for the RGS and the IBIS/ISGRI spectrum, respectively. The effective exposure time of the spectrum was of $38.2 \mathrm{ks}$ for the EPIC, $12.0 \mathrm{ks}$ for the RGS, and $17.6 \mathrm{ks}$ for IBIS/ISGRI. The averaged flux is $4.3 \times 10^{-11} \mathrm{erg} \mathrm{cm}^{-2} \mathrm{~s}^{-1}(1-10 \mathrm{keV})$. We note that the value of $N_{\mathrm{H}}^{\text {los }}$ in the partial covering model is compatible with the expected Galactic value in the direction of the source (Dickey \& Lockman 1990).

To investigate possible spectral changes during the $X M M$ Newton observation, we extracted different EPIC-pn and EPICMOS spectra of the source by choosing the time intervals corresponding to the most noticeable variations of the HR. The different spectral intervals are labeled with letters $\mathrm{A}-\mathrm{Z}$ and are color-coded in Fig. 1 (top). Spectra A-T were characterized by relatively low statistics and could be well fit by using a simple absorbed power-law model. Spectrum $\mathrm{Z}$ was extracted by using a significantly longer exposure time and could be fit only by using a partial covering cutoff power-law model. We fixed the cutoff energy at $31.7 \mathrm{keV}$ (based on the INTEGRAL results) and measured $N_{\mathrm{H}}^{\text {los }}=(2.4 \pm 0.2) \times 10^{22} \mathrm{~cm}^{-2}$, $N_{\mathrm{H}}=(9.5 \pm 2.2) \times 10^{22} \mathrm{~cm}^{-2}, f=0.57 \pm 0.05, \Gamma=1.4 \pm 0.1$, and an average flux of $1.4 \times 10^{-11} \mathrm{erg} \mathrm{cm}^{2} \mathrm{~s}^{-1}$. For each selected time interval (A to Z), the EPIC-pn and EPIC-MOS spectra were fit simultaneously and the estimated values of the absorption column density, power-law photon index, and flux are summarized in Table A.1. Some results are also plotted in Fig. 1 (top). These fits revealed only a moderate change of the absorption column density (a factor of $\sim 2$ ) and power-law photon index despite the remarkable variation in the X-ray flux (a factor of $\gtrsim 10$ ). 

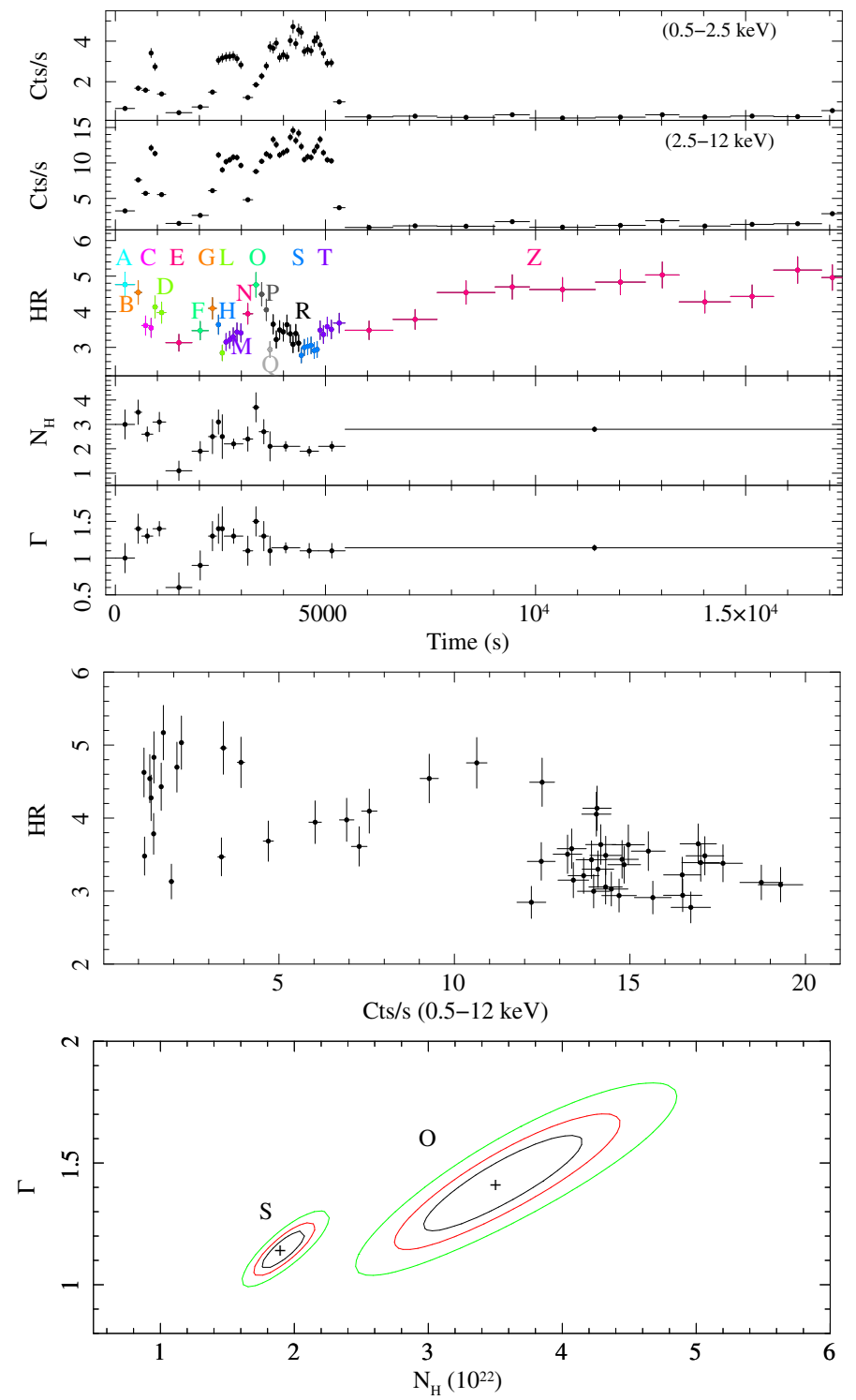

Fig. 1. Results obtained from observation 0306170401 of IGR J184500435. Top: EPIC-pn light curve of the source in two energy bands and the corresponding HR (an adaptive rebinning was used to achieve a minimum $S / N>15$ in each soft time bin). The letters in the middle panel indicate the different HR resolved spectra listed in Table A.1. The last two panels show the results obtained from the spectral fits to the HRresolved spectral analysis (the measured absorption column density in units of $10^{22} \mathrm{~cm}^{-2}$ and the photon index $\left.\Gamma\right)$. Middle: hardness intensity diagram (HID). Bottom: contour plot showing the ranges spanned by the power-law photon index and the absorption column density measured from the most relevant time intervals for the spectral variations marked in the second plot from the top (in this case the intervals " $O$ " and "S").

\subsubsection{Observation 0728370801}

This observation was not affected by flaring background intervals and thus the full exposure time could be retained for the scientific analysis. The pn was operated in small window mode, while the two MOS were set in large window mode. As this observation has not yet been reported elsewhere, we show the fullband EPIC-pn light curve in Fig. 3, together with the energyresolved light curves, the HR, and the HID.

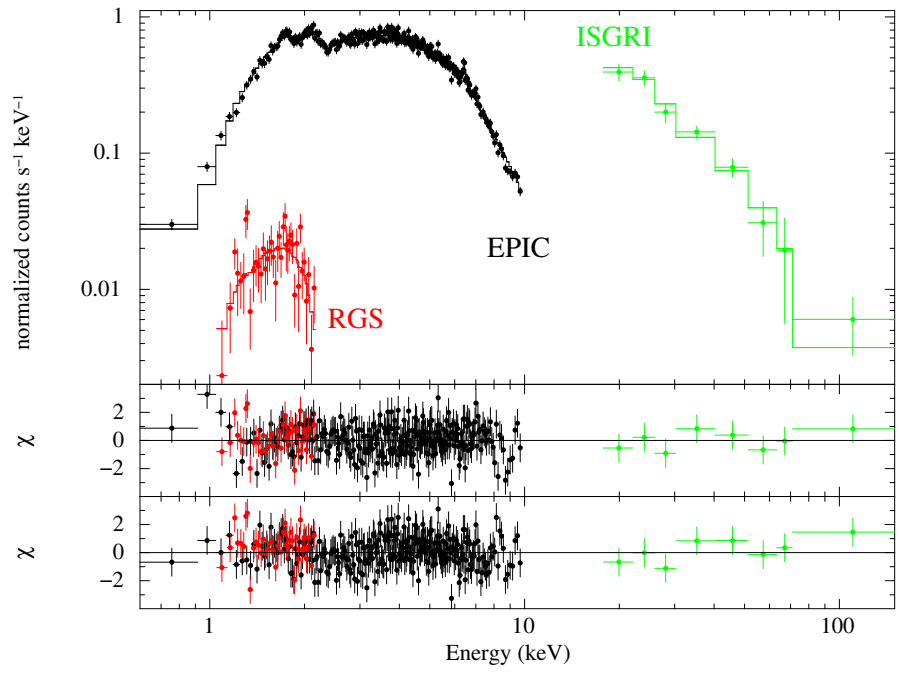

Fig. 2. Broadband X-ray spectrum of IGR J18450-0435 obtained by summing the data from the three XMM-Newton EPIC cameras in observation 0306170401, combining the data from the two RGSs in the same observation, and using the INTEGRAL data described in the text. The best fit is obtained with a partial covering model comprising a cutoff power law and an iron line. The middle panel shows the residuals from this fit. The bottom panel shows the residuals obtained by fitting the spectrum with the model proposed by Zurita Heras \& Walter (2009).

The source displayed two separated periods of flaring activity. During the first, a bright episode was accompanied by lower intensity peaks, and lasted roughly $5 \mathrm{ks}$ in total. The second flare appeared isolated and lasted less than $1 \mathrm{ks}$. We extracted first the average source spectra for all EPIC cameras using the entire exposure time available. We verified with the EPAPLOT tool that the pn was not affected by pileup and thus we used the flux measured by this instrument to correct the pileup on the two MOS. Due to the high extinction measured in the direction of the source (see below), the reduction of the two RGSs data did not result in usable scientific products. The average spectrum of the source cannot be satisfactorily fit by using a single powerlaw model $\left(\chi_{\text {red }}^{2}=1.39 / 318\right)$. As in observation 0306170401 (see Sect. 2.1.1), we improved the results by using a model comprising a cutoff power law (the cutoff energy was fixed to $31.7 \mathrm{keV}$ ) and a partial absorber (PCFABS in XSPEC). Using the same notations as in Sect. 2.1.1, we measured $N_{\mathrm{H}}^{\text {los }}=\left(2.7_{-1.3}^{+1.0}\right) \times 10^{22} \mathrm{~cm}^{-2}$, $N_{\mathrm{H}}=\left(6.6_{-0.8}^{+1.2}\right) \times 10^{22} \mathrm{~cm}^{-2}, f=0.83_{-0.13}^{+0.09}, \Gamma=1.1 \pm 0.1$, $F=1.1 \times 10^{-11} \mathrm{erg} \mathrm{cm}^{-2} \mathrm{~s}^{-1}, C_{\mathrm{MOS} 1}=1.12 \pm 0.04, C_{\mathrm{MOS} 2}=$ $1.05 \pm 0.03$, and $\chi_{\text {red }}^{2} /$ d.o.f. $=1.11 / 316$.

Following the HR variations, we also extracted the eight different spectra indicated in Fig. 3 (second plot from the top). The HID does not show any significant correlation, but similarly to the case of observation 0306170401, it is possible to identify intervals of count-rates corresponding to higher/lower HR values than average. Due to the lower statistics, all the HR-resolved spectra could be well fit by using a simple absorbed power-law model and we present all the results in Table A.1. Some of the results are also plotted in Fig. 3 (second plot from the top). The largest variation in the column density is found between spectra $\mathrm{C}$ and $\mathrm{G}$, which correspond to the rise to the first large flare and the time interval before the second. The contour plots in Fig. 3 (bottom) shows that the variation is highly significant and cannot be associated with the degeneracy between the spectral slope and the absorption column density. 

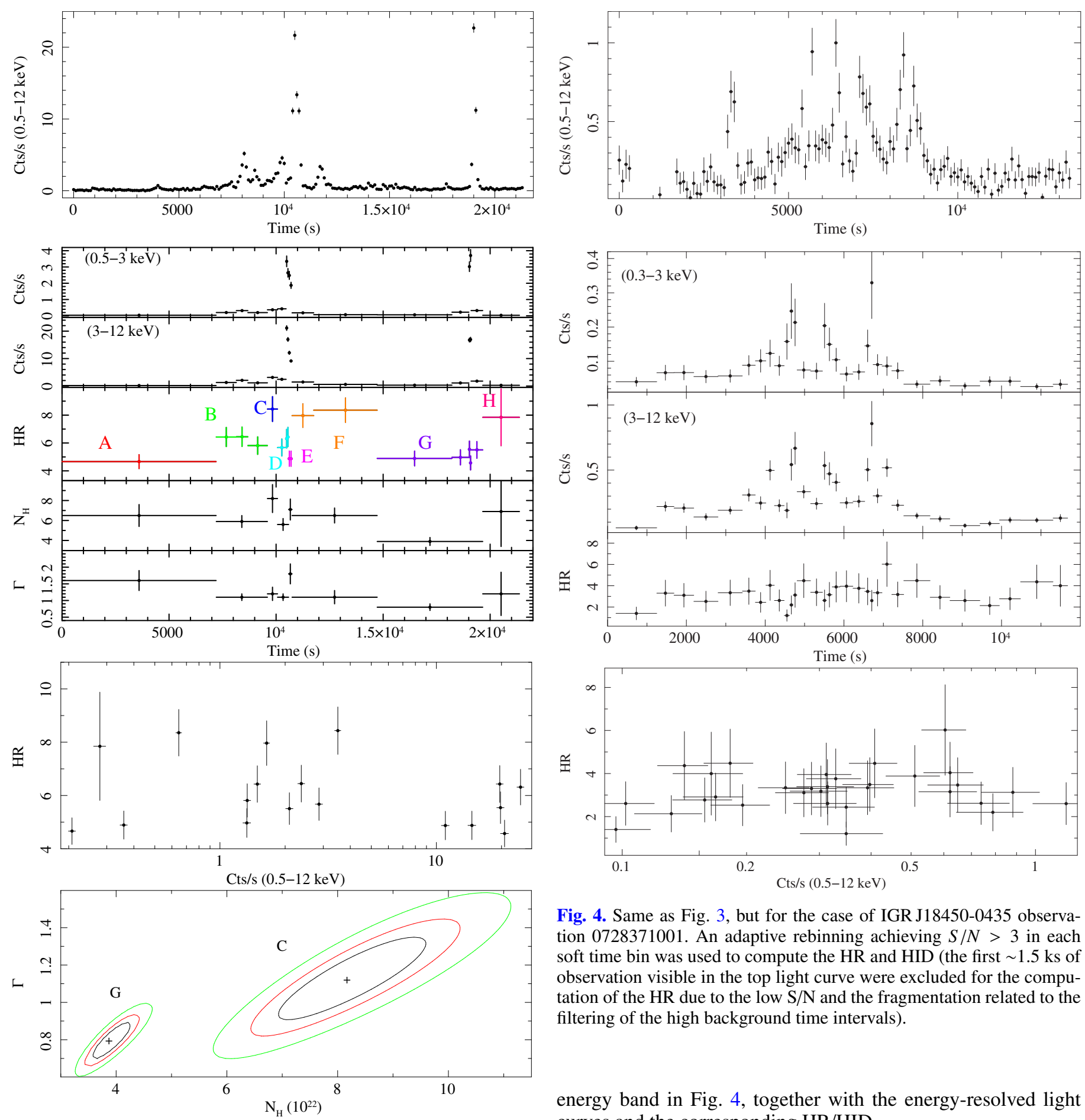

Fig. 3. Same as Fig. 1, but for IGR J18450-0435 observation 0728370801 . As this observation was not reported previously in the literature, the source EPIC-pn light curve in the full energy range $(0.5-12 \mathrm{keV})$ is also shown on the top plot (time bin $100 \mathrm{~s})$. An adaptive rebinning achieving $S / N>10$ in each soft time bin was used to compute the HR and HID.

\subsubsection{Observation 0728371001}

In this observation the same instrument setup as in 0728370801 was used, and the presence of solar flares led to a reduced effective exposure time of $8.5 \mathrm{ks}$ for the EPIC-pn and $6.9 \mathrm{ks}$ for the two MOS. The observation has not yet been reported elsewhere, and thus we show the EPIC-pn light curve in the full available

Fig. 4. Same as Fig. 3, but for the case of IGR J18450-0435 observation 0728371001. An adaptive rebinning achieving $S / N>3$ in each soft time bin was used to compute the HR and HID (the first $\sim 1.5 \mathrm{ks}$ of observation visible in the top light curve were excluded for the computation of the HR due to the low $\mathrm{S} / \mathrm{N}$ and the fragmentation related to the filtering of the high background time intervals).

energy band in Fig. 4, together with the energy-resolved light curves and the corresponding HR/HID.

The source displayed low level X-ray flaring activity, but no significant HR variation could be seen (see Fig. 4). This is also confirmed by the HID in which all points are consistent with each other (within the uncertainties). Consequently, we report for completeness on the average X-ray spectrum extracted from the three EPIC cameras, but we did not perform any HRresolved spectral analysis. The best fit to the average spectra was achieved by using a simple absorbed power-law model. We measured an absorption column density of $(3.9 \pm 0.6) \times 10^{22} \mathrm{~cm}^{-2}$ and a power-law photon index of $1.4 \pm 0.2$. The normalization constant of the MOS1 (MOS2) with respect to the pn was of $1.1 \pm 0.1(0.96 \pm 0.09)$. The average $1-10 \mathrm{keV} X$-ray flux measured from the spectral fit is $2.5 \times 10^{-12} \mathrm{erg} \mathrm{cm}^{-2} \mathrm{~s}^{-1}$ (not corrected for absorption). 
E. Bozzo et al.: The accretion environment of supergiant fast X-ray transients probed with XMM-Newton
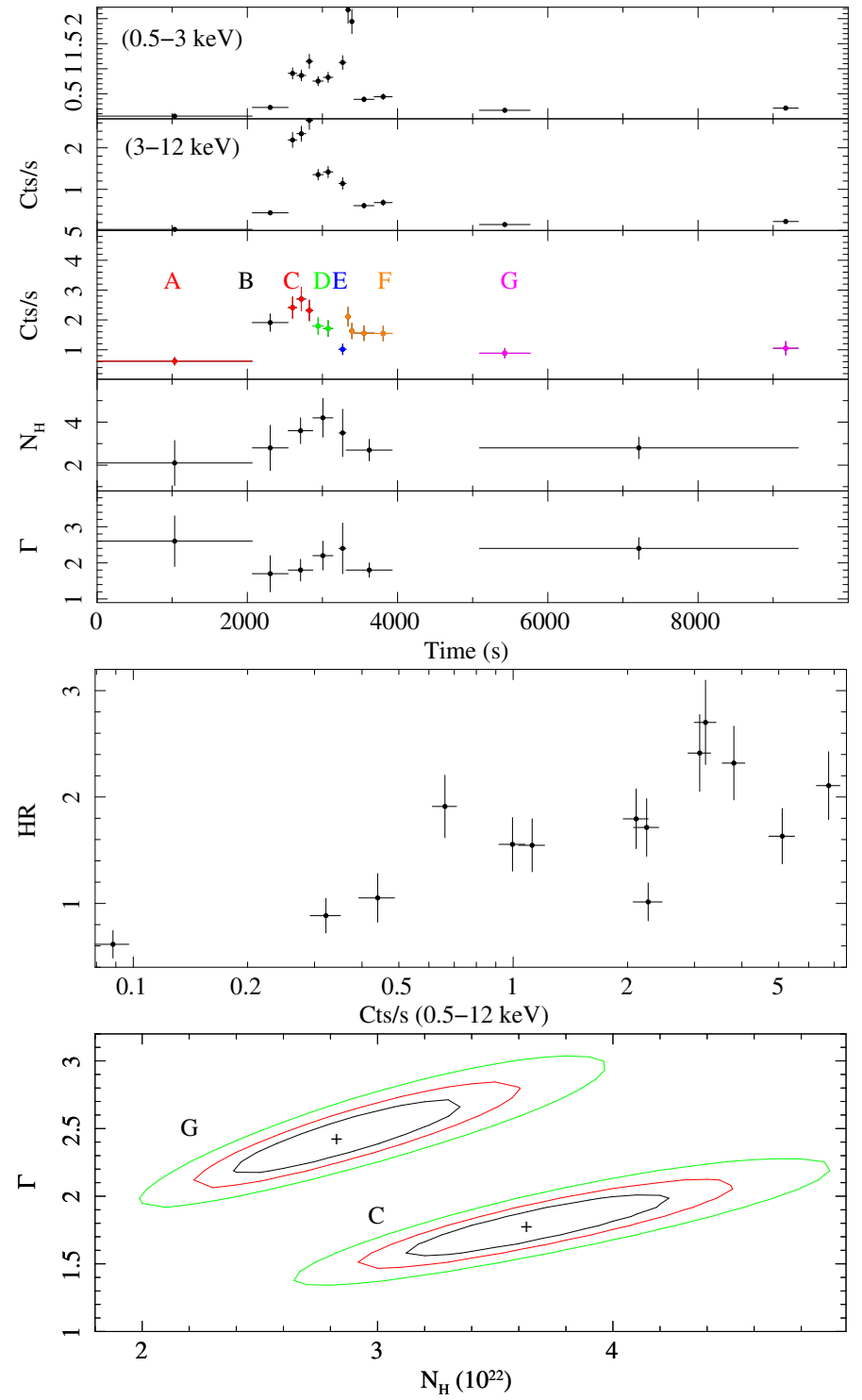

Fig. 5. Same as Fig. 1, but for the case of IGR J17544-2619 observation 0148090501. An adaptive rebinning achieving $S / N>10$ in each soft time bin was used to compute the HR and HID. The most relevant time intervals for the spectral variations are in this case "C" and " $\mathrm{G}$ ".

\subsection{IGRJ17544-2619}

\subsubsection{Observation 0148090501}

This observation was originally reported by González-Riestra et al. (2004), who retained for the scientific analysis the entire exposure time available. During our re-analysis of the data, we found that about $\sim 40 \%$ of the total exposure time was significantly affected by a heavily flaring background and thus, based on the presently available suggestions in the XMM-Newton data analysis threads, we discarded the corresponding time intervals for further analysis. One structured flare was recorded from the source during a time interval where the background was low and stable. The energy-resolved light curves of IGR J17544-2619 are shown in Fig. 5, together with the corresponding HR and HID.

Due to the relatively low $\mathrm{S} / \mathrm{N}$ below $3 \mathrm{keV}$, no RGS spectra could be extracted from this observation. The summed EPICpn, MOS1, and MOS2 average spectra could be well fit $\left(\chi_{\text {red }}^{2}=\right.$ $1.0 / 150)$ by using a simple absorbed power-law model. We measured $N_{\mathrm{H}}=(2.9 \pm 0.2) \times 10^{22} \mathrm{~cm}^{-2}$ and $\Gamma=1.95 \pm 0.11$. The

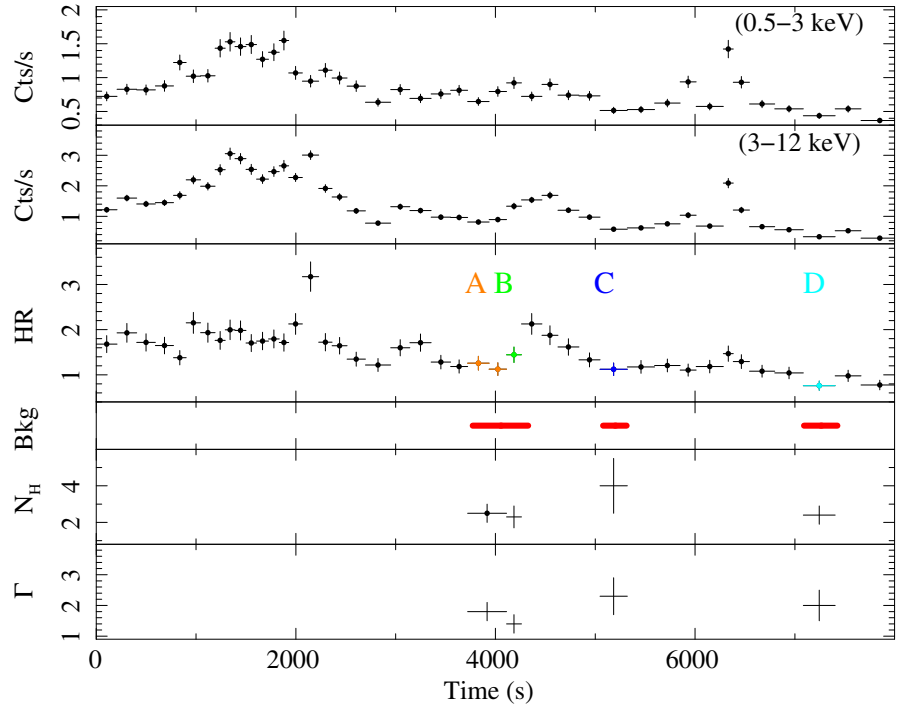

Fig. 6. Same as Fig. 1, but for the case of IGR J17544-2619 observation 0154750601. An adaptive rebinning achieving $S / N>11$ in each soft time bin was used to compute the HR before filtering the light curves for the high flaring background. Only the time intervals marked with a red solid line in the fourth panel from the top could be used for the spectral analysis, due to the high flaring background that affected the rest of the observation.

absorbed 1-10 keV flux is $5.5 \times 10^{-12} \mathrm{erg} \mathrm{cm}^{-2} \mathrm{~s}^{-1}$ (effective exposure time $6.6 \mathrm{ks}$ ).

To investigate the source spectral variability during the flare, we extracted six spectra corresponding to the HR changes in Fig. 5. They could all be well fit with a simple absorbed power-law model and we present the results in Table A.2. The largest change in the source spectral parameters was observed between the rise to the flare (interval C) and the post flare spectrum (interval G). The latter has a similar HR to the pre-flare interval A, but also a higher statistics. This result is also confirmed by the HID, which shows that the highest HR is not achieved at the highest intensity. The contour plots of the spectra $\mathrm{C}$ and $\mathrm{G}$ suggested that the power-law photon index was harder during the rise to the flare, accompanied by a likely increase in the absorption column density. We note that the flux of the source before the flare was a factor of $\sim 5$ lower than that achieved at the end of the flare. The relatively poor statistics of the A spectrum do not allow for a more refined assessment of possible spectral changes before and after the flare.

\subsubsection{Observation 0154750601}

The EPIC-pn and the MOS1 were operated in timing mode, while the MOS2 was in full frame. The total exposure available was $\sim 8 \mathrm{ks}$ for the two MOS cameras and $2.5 \mathrm{ks}$ for the EPICpn (the EPIC-pn started observing the target about $5.5 \mathrm{ks}$ after the two MOS cameras). González-Riestra et al. (2004) were the fist to report on these data and retained for the scientific analysis the entire exposure time available. During our re-analysis of the data, we found that the observation was heavily affected by the flaring background. The background filtering technique suggested in the currently available XMM-Newton data analysis threads would result in a nearly complete rejection of all data, although the source displayed relatively bright flares during this observation. As the flaring background in the EPIC cameras is due to solar protons arriving onto the $\mathrm{X}$-ray detectors with hardly predicable spectral properties, it is not possible to correct the 
distortion in energy of the source spectra extracted during the affected time intervals ${ }^{5}$. The only data that can be safely used for the scientific analysis are thus those collected during the time intervals marked with a red solid line in Fig. 6 (bottom panel). We carried out the spectral analysis only during these time intervals. Due to the fragmented exposure, we did not compute the HID for this observation. The average MOS1 and MOS2 spectra (effective exposure time $1.8 \mathrm{ks}$ ) fit simultaneously with an absorbed power-law model provided the most accurate description of the source average X-ray emission. We measured $N_{\mathrm{H}}=(2.2 \pm$ $0.2) \times 10^{22} \mathrm{~cm}^{-2}$ and $\Gamma=1.7 \pm 0.1\left(\chi_{\text {red }}^{2} /\right.$ d.o.f. $\left.=1.25 / 138\right)$. The absorbed $1-10 \mathrm{keV}$ flux is $2.4 \times 10^{-11} \mathrm{erg} \mathrm{cm}^{-2} \mathrm{~s}^{-1}$. The fit to the average EPIC-pn spectrum (exposure time $1.2 \mathrm{ks}$ ) gave a consistent absorption column density $\left(N_{\mathrm{H}}=(2.6 \pm 0.4) \times 10^{22} \mathrm{~cm}^{-2}\right)$ and a slightly steeper power-law photon index of $\Gamma=2.1 \pm 0.2$ $\left(\chi_{\text {red }}^{2} /\right.$ d.o.f. $\left.=1.28 / 64\right)$. This is in agreement with the lower HR visible in Fig. 6 toward the end of the observation (where the EPIC-pn data are available).

We then extracted four source spectra during the usable time intervals following the HR variations, as shown in Fig. 6 (the pn data were only available during the time interval D). We present the results of the fits to the spectra A-D in Table A.2 (some are also plotted in Fig. 6). No significant variations of the spectral parameters could be revealed due to the large associated uncertainties.

\subsubsection{Observation 0679810401}

The two MOS were operated in full frame, while the EPIC-pn was set in small window mode. No flaring background intervals were recorded. These data were first presented by Drave et al. (2014). The energy-resolved EPIC-pn light curves of the source are shown in Fig. 7, together with the corresponding HR and HID. The source displayed only a marginal flaring activity toward the end of the observation. We combined the three EPIC spectra and found that the average spectrum of the source could not be well described by a single absorbed power-law model $\left(\chi_{\text {red }}^{2}=1.47 / 111\right)$. We adopted a partial covering model for the spectral fit and we measured $N_{\mathrm{H}}^{\mathrm{los}}=(1.3 \pm 0.2) \times 10^{22} \mathrm{~cm}^{-2}$, $N_{\mathrm{H}}=\left(3.4_{-0.8}^{1.0}\right) \times 10^{22} \mathrm{~cm}^{-2}, f=0.73 \pm 0.08$, and $\Gamma=$ $2.3 \pm 0.2$. The average (absorbed) $1-10 \mathrm{keV} \mathrm{X-ray} \mathrm{flux} \mathrm{is} 2.1 \times$ $10^{-12} \mathrm{erg} \mathrm{cm}^{-2} \mathrm{~s}^{-1}$.

We extracted five spectra following the HR variations in Fig. 7 and give the results of the fits to these spectra in Table A.2. The relevant contour plots obtained from the spectral fits are shown in Fig. 7 (bottom). We found an indication of a slight increase in the absorption column density and hardening of the power law during the rise to the faint flare (spectra B and D). This is also confirmed by the HID (Fig. 7, middle) which shows that the source emission becomes harder at count-rates $\gtrsim 0.5 \mathrm{cts} \mathrm{s}^{-1}$.

\section{3. $S A X J 1818.6-1703$}

\subsubsection{Observation 0693900101}

This is the only available observation where the source was pointed and detected by XMM-Newton (see also Bozzo et al. 2008a, 2012). A first analysis of these data was reported by Boon et al. (2016). During our re-analysis of the data, we noticed that the observation was affected by a high background

\footnotetext{
5 http://xmm.esac.esa.int/external/xmm_user_support/ documentation/uhb/epicextbkgd.html
}
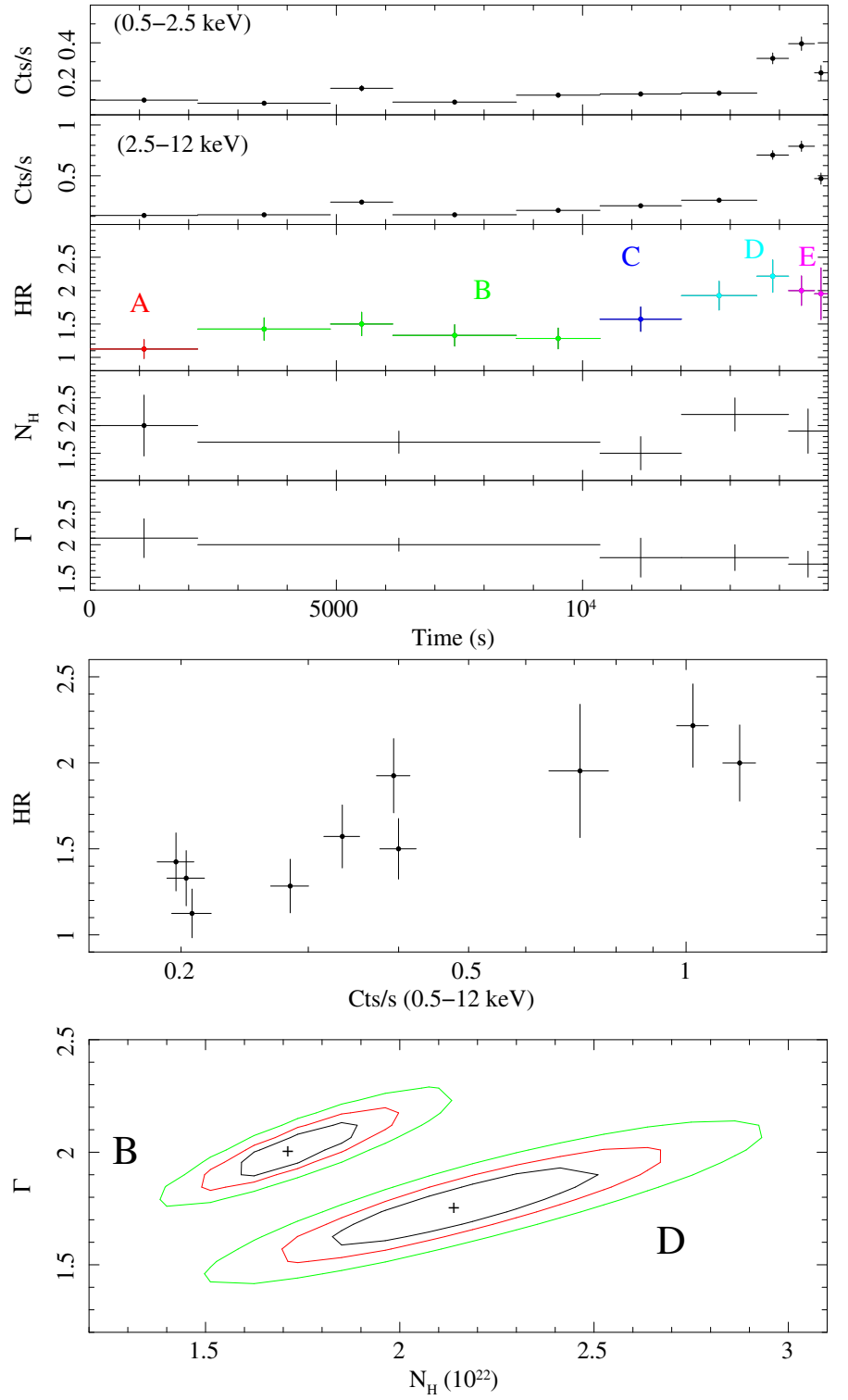

Fig. 7. Same as Fig. 1, but for the case of IGR J17544-2619 observation 0679810401 . An adaptive rebinning achieving $S / N>11$ in each soft time bin was used to compute the HR and HID.

during the last $8.3 \mathrm{ks}$, and thus we discarded these data for the spectral analysis. The EPIC-pn was operated in small window, while the two MOS were operated in full frame. Given the relatively high count-rate of the source after the initial $\sim 15 \mathrm{ks}$, the MOS suffered significant pileup, and thus we discarded these data (the quality of the pn data was high enough to carry out all necessary investigations). The average EPIC-pn spectrum (effective exposure time $15.6 \mathrm{ks}$ ) could be fit with a simple absorbed power-law model. We measured $N_{\mathrm{H}}=(27.0 \pm 1.1) \times 10^{22} \mathrm{~cm}^{-2}$, $\Gamma=0.48 \pm 0.06$, and a flux of $3.5 \times 10^{-11} \mathrm{erg} \mathrm{cm}^{-2} \mathrm{~s}^{-1}$ in the $1-10 \mathrm{keV}$ energy range. As the source is heavily absorbed, we did not attempt an extraction of the RGS data.

We extracted the adaptively rebinned EPIC-pn light curves of SAX J1818.6-1703 and computed the source HR/HID shown in Fig. 8. We extracted a number of HR resolved spectra following the HR variations. Simultaneous MOS1 and MOS2 spectra were only extracted for the time intervals A, B, C, and D to avoid pileup issues. We fit all EPIC spectra with an absorbed powerlaw model, which provided a good description of the data in all 
E. Bozzo et al.: The accretion environment of supergiant fast X-ray transients probed with XMM-Newton
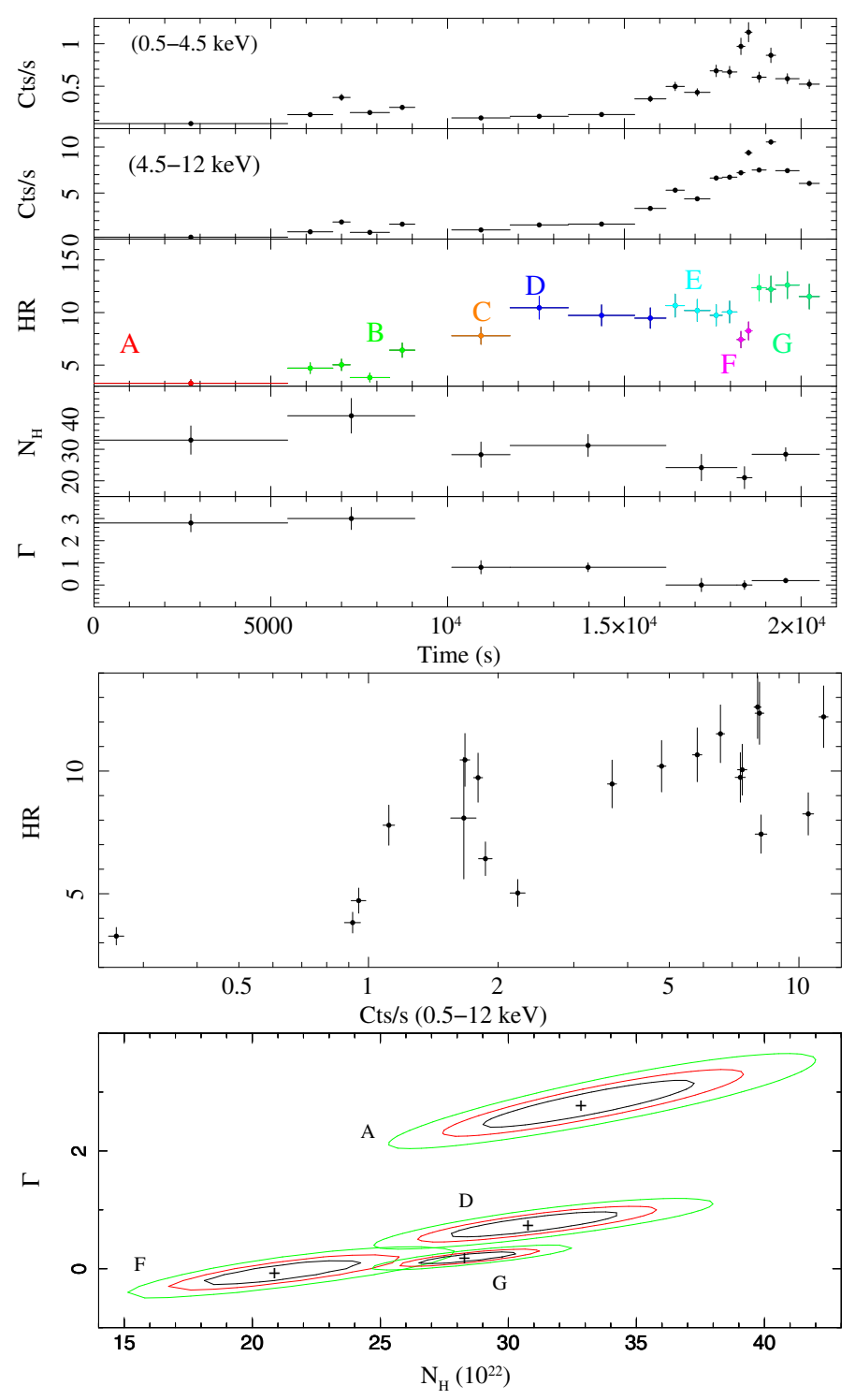

Fig. 8. Same as Fig. 1, but for the case of SAX J1818.6-1703 observation 0693900101. An adaptive rebinning achieving $S / N>12$ in each soft time bin was used to compute the HR and HID.

cases. The results are summarized in Table A.3 and partly plotted in Fig. 8. We recorded a progressive hardening of the X-ray emission during the rise to the flare (time intervals $\mathrm{A}, \mathrm{B}, \mathrm{C}, \mathrm{D}$, and $\mathrm{E}$ ) and a significant decrease in the absorption column density at the peak of the event (time interval F). The data also show some evidence of a re-increase of the $N_{\mathrm{H}}$ following the peak of the flare. Remarkable changes were also observed in the powerlaw spectral index, as suggested by the HID (where the source emission becomes harder at higher count-rates). In order to further confirm these findings, we also show in Fig. 8 the relevant contour plots of the fit parameters derived from the analysis of the HR-resolved spectra.

\subsection{IGRJ17354-3255}

\subsubsection{Observation 0693900201}

During this observation, not yet reported in other works, the EPIC-pn was operated in small window mode, but unfortunately it was pointed to a different nearby source and thus the collected data are not usable. The two MOS cameras were instead operated in full frame and thus included IGR J17354-3255 in their field of view. In the following, we only report on the MOS data analysis and we merge in all cases the MOS1 and MOS2 spectra by using the SAS tool EPICSPECCOMBINE to improve the statistics. The observation was affected by a moderately high background for the first $\sim 3 \mathrm{ks}$ and by very high background during the last $\sim 6 \mathrm{ks}$. We retained the first $3 \mathrm{ks}$ of the observation as bright flares from the source were also detected, and discarded the last part of the observation for the scientific analysis. The average spectrum of the source is shown in Fig. 10 as an example (which looks similar to the spectra extracted from the following two observations 0701230101 and 0701230701). An acceptable fit to this spectrum $\left(\chi_{\text {red }}^{2} /\right.$ d.o.f. $\left.=0.87 / 146\right)$ could be obtained by using an absorbed power law and a neutral iron emission line. We measured $N_{\mathrm{H}}=(10.2 \pm 0.2) \times 10^{22} \mathrm{~cm}^{-2}, \Gamma=1.34 \pm 0.04$, $E_{\mathrm{Fe}}=6.36 \pm 0.02 \mathrm{keV}, E Q W_{\mathrm{Fe}}=0.06 \mathrm{keV}$, and a flux of $3.2 \times 10^{-11} \mathrm{erg} \mathrm{cm}^{-2} \mathrm{~s}^{-1}$ in the $1-10 \mathrm{keV}$ energy range (effective exposure time $27.6 \mathrm{ks}$ ).

The energy-resolved light curves of the source are shown in Fig. 9, together with the corresponding HR and HID. To investigate the variability suggested by the changes in the HR across the observation we extracted the HR-resolved spectra A-E indicated in the same figure. All spectra could be well fit with an absorbed power law and we present all results in Table A.4. Some of the results obtained from the HR-resolved spectral analysis are also shown in Fig. 9, together with the contour plots of the relevant spectral parameters determined from the fits. The most noticeable changes in the source spectral parameters are recorded during the lower X-ray intensity period (interval C) separating the flares at the beginning and at the end of the observation. This is also clearly visible from the HID, which shows that the highest HR values are achieved at the count-rate specifically recorded toward the middle of the low X-ray intensity period.

\subsubsection{Observation 0701230101}

During this observation, not yet reported in other literature works, the three EPIC cameras were operated in large window mode and several time intervals affected by a high background were recorded. The merged EPIC-pn and MOS spectrum extracted by using all available exposure time $(17.1 \mathrm{ks})$ could be well fit $\left(\chi_{\text {red }}^{2} /\right.$ d.o.f. $\left.=1.11 / 667\right)$ with an absorbed power-law model, plus a neutral iron emission line. We measured $N_{\mathrm{H}}=$ $(7.4 \pm 0.2) \times 10^{22} \mathrm{~cm}^{-2}, \Gamma=1.40 \pm 0.04, E_{\mathrm{Fe}}=6.43 \pm 0.02 \mathrm{keV}$, $E Q W_{\mathrm{Fe}}=0.04 \mathrm{keV}$, and a flux of $1.1 \times 10^{-11} \mathrm{erg} \mathrm{cm}^{-2} \mathrm{~s}^{-1}$ in the 1-10 keV energy range.

After having excluded the affected time intervals, we obtained the energy-resolved light curves displayed in Fig. 11, together with the corresponding HR and HID. A number of moderately bright flares were recorded from the source. We extracted the source A-M spectra during the HR variations as indicated in Fig. 11 and fit them with an absorbed power-law model. The results are summarized in Table A.4 and some are also plotted in Fig. 11. We recorded a significant increase in the absorption column density preceding the two flares (time intervals B and E) and a drop close to their peaks (time intervals $\mathrm{C}$ and $\mathrm{F}$ ).

\subsubsection{Observation 0701230701}

During this observation, not reported yet in other works, the EPIC cameras were operated in large window mode and no high background time intervals were recorded. We could thus 

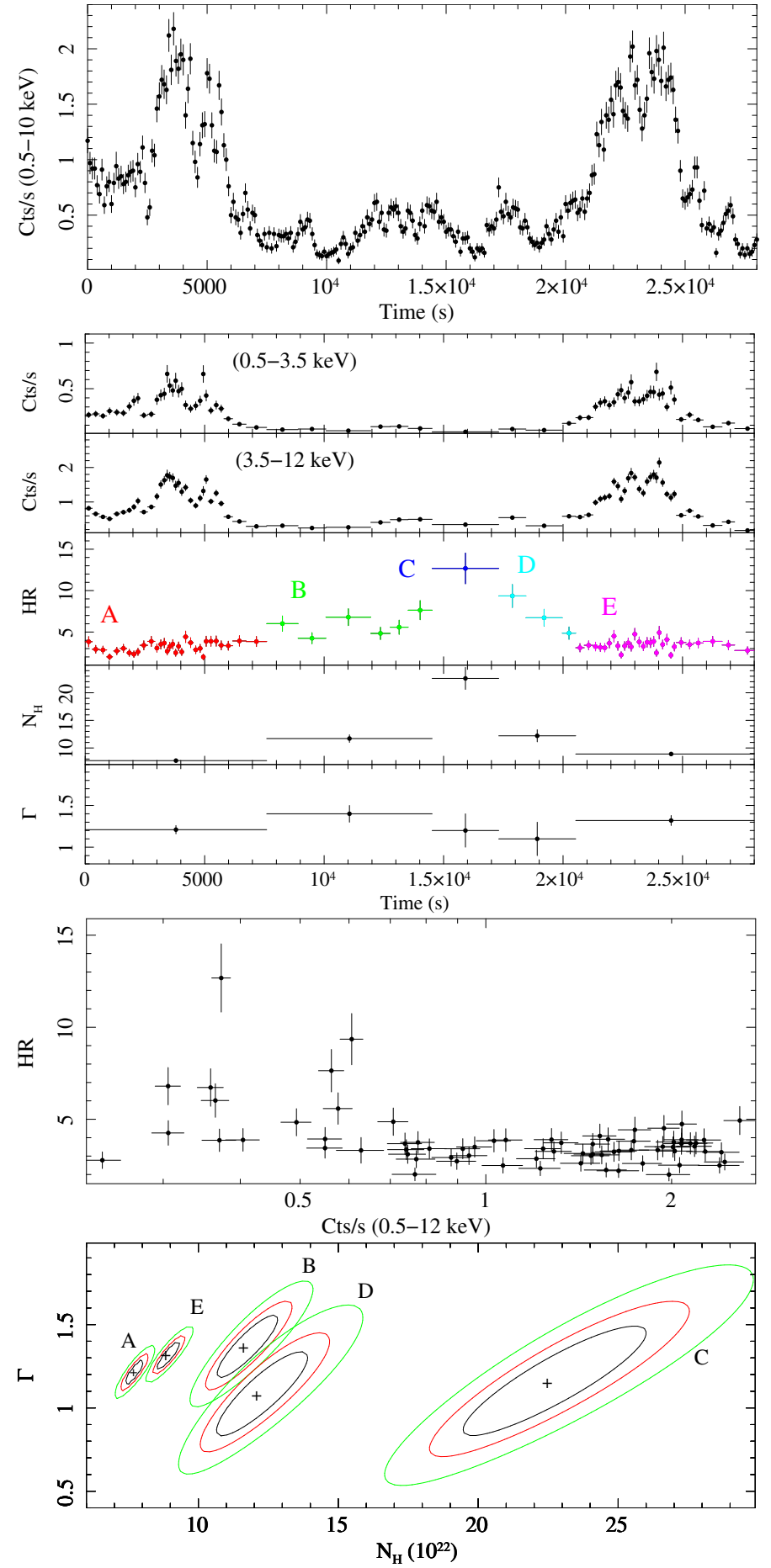

Fig. 9. Same as Fig. 3, but for the case of IGR J17354-3255 observation 0693900201. An adaptive rebinning achieving $S / N>7$ in each soft time bin was used to compute the HR and HID.

retain the entire exposure time available for the scientific analysis. The average spectrum obtained by merging all EPIC cameras (effective exposure time $19.1 \mathrm{ks}$ ) could be well fit $\left(\chi_{\text {red }}^{2} /\right.$ d.o.f. $\left.=1.11 / 667\right)$ with an absorbed power-law model plus an iron emission line. We measured in this case $N_{\mathrm{H}}=(6.0 \pm$ $0.1) \times 10^{22} \mathrm{~cm}^{-2}, \Gamma=1.28 \pm 0.03, E_{\mathrm{Fe}}=6.41 \pm 0.02 \mathrm{keV}$, $E Q W_{\mathrm{Fe}}=0.04 \mathrm{keV}$, and a flux of $1.0 \times 10^{-11} \mathrm{erg} \mathrm{cm}^{-2} \mathrm{~s}^{-1}$ in the 1-10 keV energy range.

The source displayed a remarkable variability, with moderately bright flares and short intervals of fainter X-ray emission.

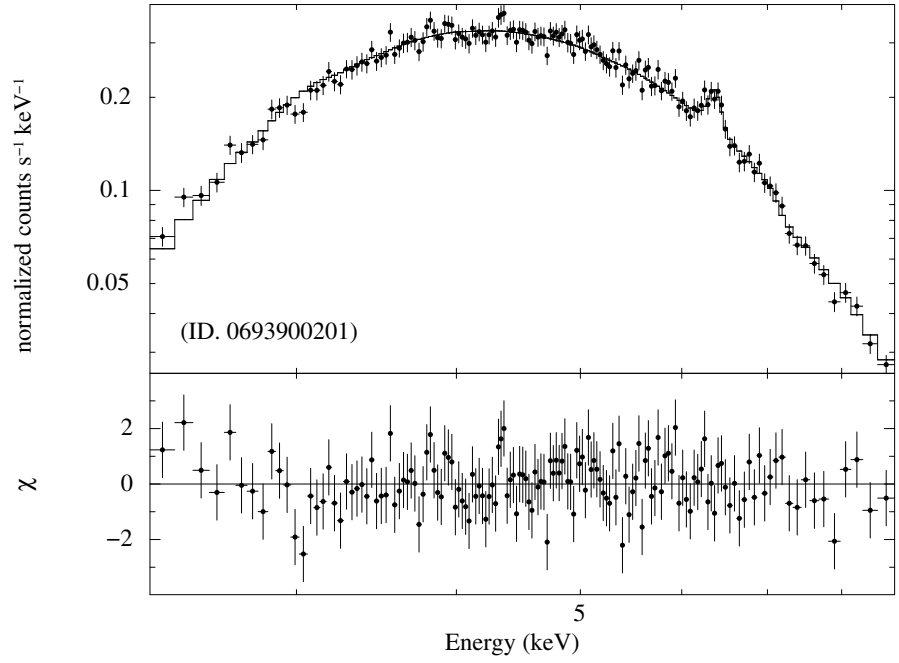

Fig. 10. Merged MOS spectra of IGR J17354-3255 in observation 0693900201 . The best fit is obtained with an absorbed power law plus a neutral iron emission line (see text for details). The residuals from the fit are shown in the bottom panel.

The energy-resolved light curves of the source are shown in Fig. 12, together with the corresponding HR and HID. At odds with the case of observation 0693900201, the most prominent HR variations were recorded during the flares. The HID shows that there is not a clear trend of the HR as a function of the X-ray intensity, but rather that the HR varies sporadically during some specific rise/decay phases of the flares. The results of the HRresolved spectral analysis are presented in Table A.4 and Fig. 12. We recorded significant increases in the absorption column density slightly before the rises of the different flares (time intervals $\mathrm{H}$ and $\mathrm{N}$ ), accompanied by moderate power-law slope variations and $N_{\mathrm{H}}$ drops at the peak of those events (time intervals $\mathrm{F}, \mathrm{L}$, and $\mathrm{O}$ ).

\subsection{IGRJ16328-4732}

\subsubsection{Observation 0679810201}

These data were first analyzed by Fiocchi et al. (2016), who found that no particularly high-flaring background time interval affected the data, and thus the entire exposure time could be used for the scientific analysis. The source displayed several relatively bright flares, achieving up to $\sim 6 \mathrm{cts} \mathrm{s}^{-1}$ in the EPICpn $(0.5-12 \mathrm{keV})$. We verified that no pileup was present in the data. The EPIC-pn energy-resolved light curves of the source are shown in Fig. 13, together with the corresponding HR and HID. A total of 16 EPIC spectra were extracted following the HR variations shown in the figure (the MOS1 and MOS2 spectra were corrected for pileup whenever required). The pn, MOS1, and MOS2 spectra were fit together with a simple absorbed powerlaw model. All results are summarized in Table A.5 and some are also plotted in Fig. 13. The largest spectral variations, especially in terms of absorption column densities, are recorded between the time interval $\mathrm{C}$ preceding the rise to the second flare and $\mathrm{E}$ or $\mathrm{N}$ (corresponding to the peaks of the two flares). The time interval $\mathrm{L}$ is also characterized by a relatively low absorption and corresponds to the separation between the second and the third flare. We note that the count-rate corresponding to these specific intervals are those for which the HID also shows a large increase/decrease in the HR (see Fig. 13). 
E. Bozzo et al.: The accretion environment of supergiant fast X-ray transients probed with XMM-Newton
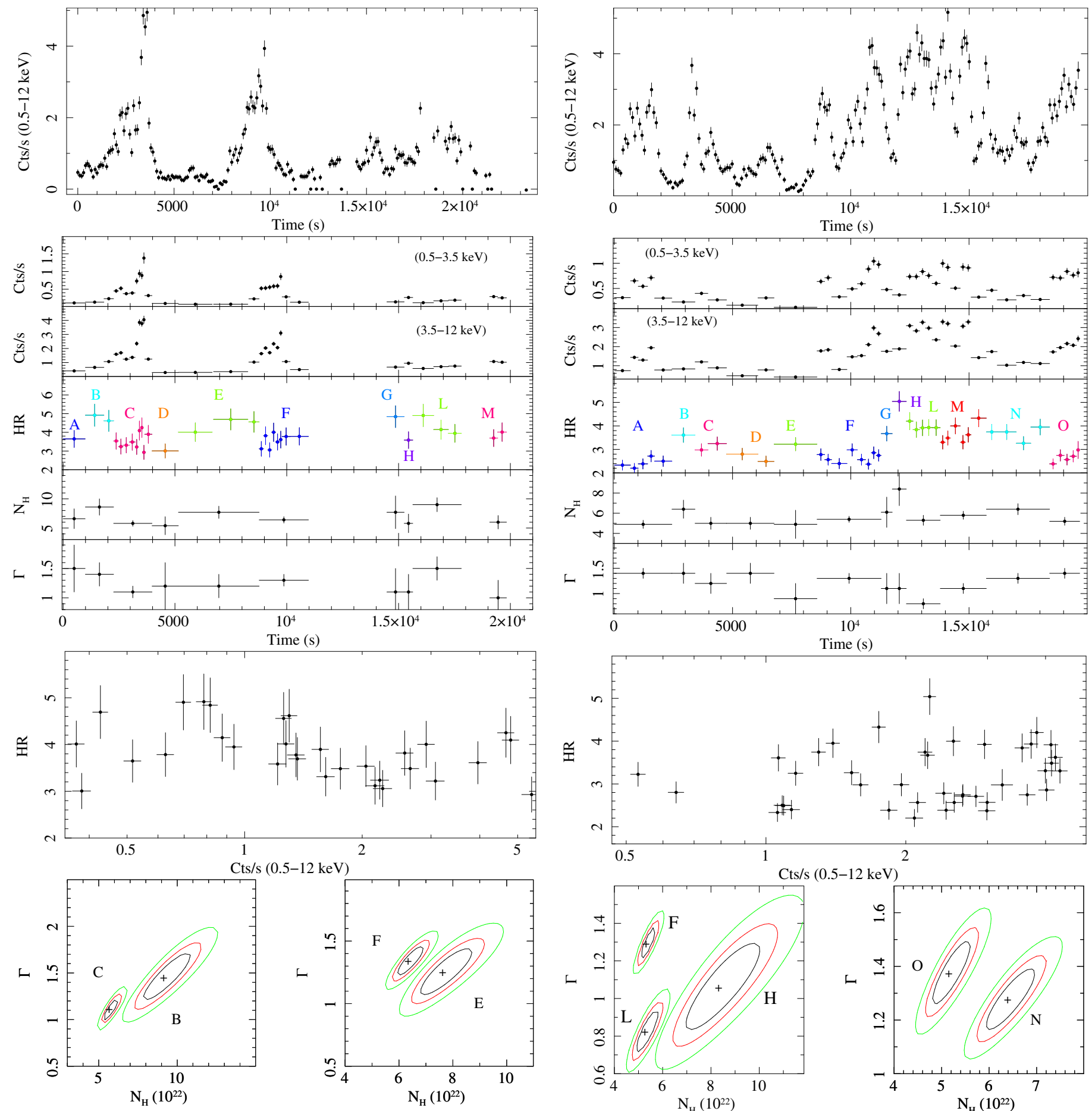

Fig. 11. Same as Fig. 3, but for the case of IGR J17354-3255 observation 0701230101 . An adaptive rebinning achieving $S / N>10$ in each soft time bin was used to compute the HR and HID.

\subsubsection{Observation 0679810301}

The analysis of these data was first discussed by Fiocchi et al. (2016). We report the source EPIC-pn energy-resolved light curves in Fig. 14, together with the corresponding HR and HID. The time intervals indicated in this figure (top) were used to extract the HR-resolved spectra for the EPIC-pn, MOS1, and MOS2 cameras. All these spectra could be well fit with a simple absorbed power-law model and we summarize all the results in Table A.5 (some results are also plotted in Fig. 14). Although the overall flaring behavior seems remarkably similar
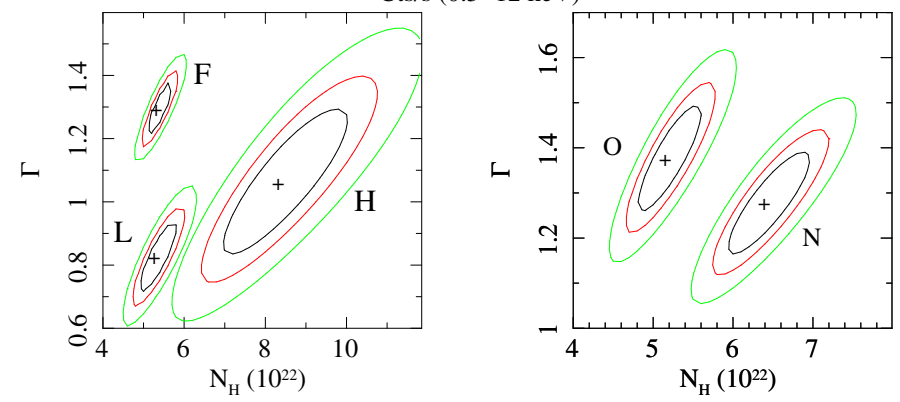

Fig. 12. Same as Fig. 3, but for the case of IGR J17354-3255 observation 0701230701. An adaptive rebinning achieving $S / N>12$ in each soft time bin was used to compute the HR and HID.

to that discussed for observation 0679810201, in the present case we measured more pronounced variations of the spectral slope (ranging from 0.3 to 1.7). The contour plots obtained from the different intervals show that in most cases there is a large degeneracy between $\Gamma$ and $N_{\mathrm{H}}$, and isolating significant changes in the absorption column density proved to be more difficult (this is also confirmed by the relatively flat HID). The largest variation of the $N_{\mathrm{H}}$ was recorded between the intervals $\mathrm{M} / \mathrm{N}$ and $\mathrm{D}$, but we were forced to merge together the data of the $\mathrm{M}$ and $\mathrm{N}$ intervals in order to improve the $\mathrm{S} / \mathrm{N}$ and obtain the results shown at the 

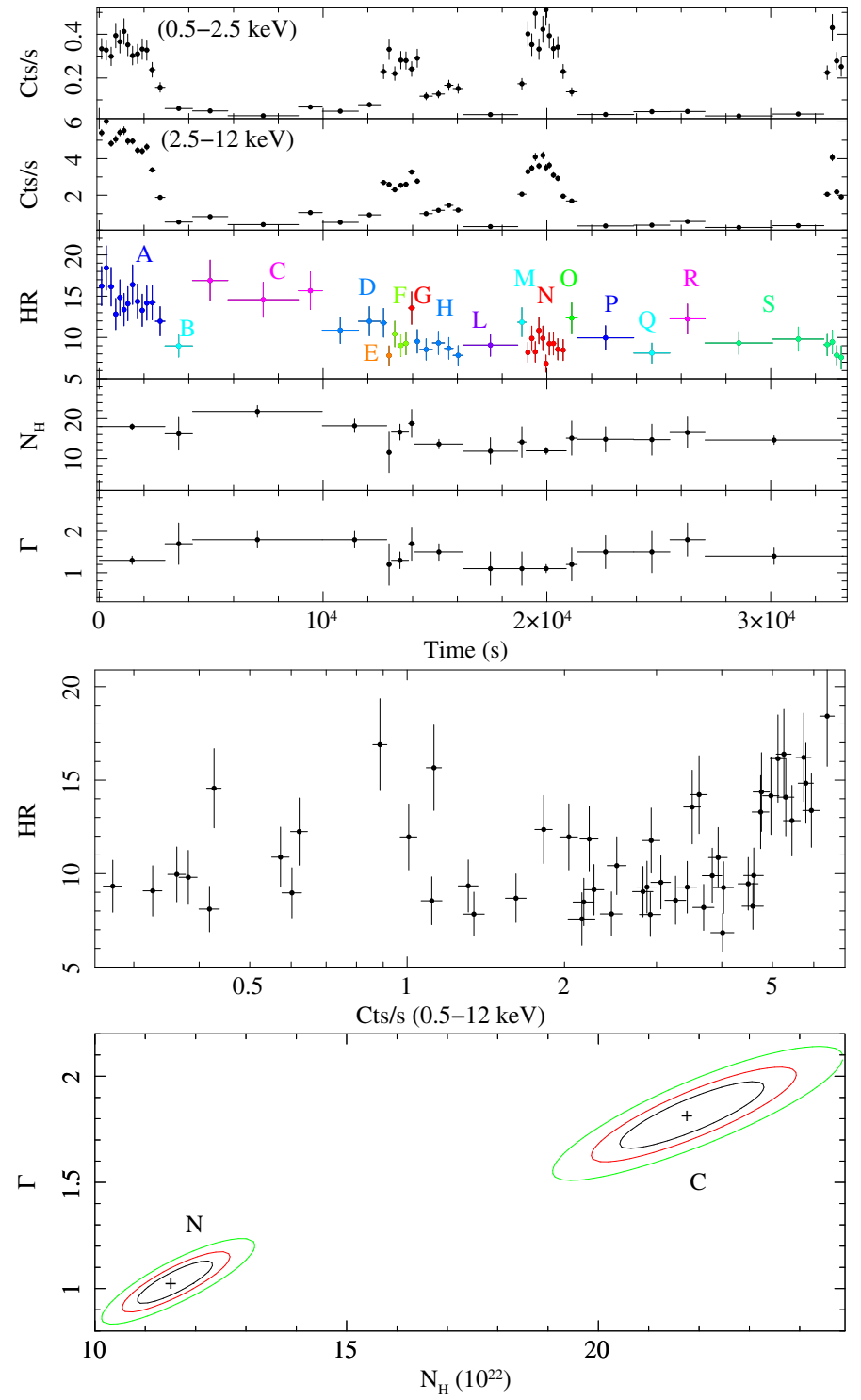

Fig. 13. Same as Fig. 1, but for the case of IGR J16328-4732 observation 0679810201. An adaptive rebinning achieving $S / N>7$ in each soft time bin was used to compute the HR and HID.

bottom of Fig. 14. The time interval D is particularly interesting because the corresponding spectra were characterized by a remarkably low absorption for which we could only set an upper limit of $<7 \times 10^{22} \mathrm{~cm}^{-2}$ (at a $90 \%$ confidence level).

\section{Overview of the results}

In this section, we summarize the most relevant findings for each of the five sources. The interpretation of these results is discussed in Sect. 4.

- IGR J18450-0435: the source displayed relatively bright flares in all three of the observations we reported on. The most prominent flares were recorded during observation 0306170401, being characterized by a flux increase of $\gtrsim 10$ with respect to the following quiescent period (the time interval Z). Despite this variability, we only measured a modest increase in the absorption column density (a factor of 2) between the flares and quiescence. We found evidence
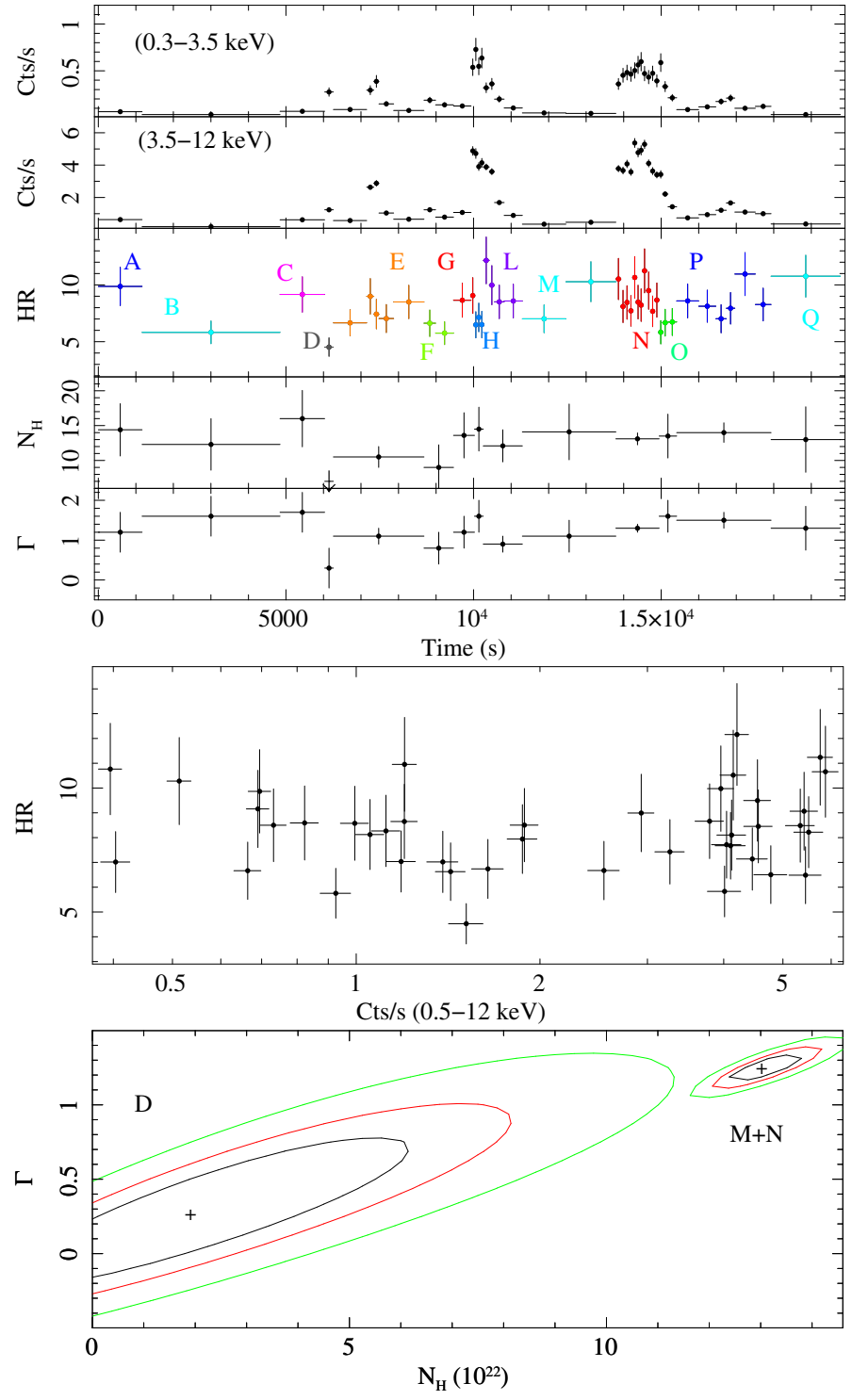

Fig. 14. Same as Fig. 1, but for the case of IGR J16328-4732 observation 0679810301 . An adaptive rebinning achieving $S / N>6$ in each soft time bin was used to compute the HR and HID.

of an increase in the absorption during the rise to the main flares (time intervals $\mathrm{A}, \mathrm{B}, \mathrm{G}$, and $\mathrm{O}$ ) and of a drop in the absorption column density every time the source approached the peak of a flare (the most significant being during the interval $\mathrm{S}$ ). The quiescent time interval $\mathrm{Z}$ is more difficult to characterize, but the fit with the partial covering model revealed a moderate but significant increase of the partial absorber column density compared to the average spectrum. A similar behavior was found during observation 0728370801 . In this case, the increase in the absorption column density before the flare was recorded during the time interval $\mathrm{C}$, while a (moderate) decrease was revealed from the fits to the intervals $D$ and $G$. We found some evidence for a re-increase in the absorption column density during the decay of the flares, i.e., between spectra $C$ and D in observation 0306170401 and between spectra $G(D)$ and $H(F)$ in observation 0728370801 .

- IGRJ17544-2619: the source showed moderately bright flares during observation 0148090501, achieving an overall dynamic range of $\gtrsim 10$ in the X-ray flux. The HR increases 
during the rise to the flare (time interval B) and drops close to the peak of the event (time interval E). We found some evidence of an increase in $N_{\mathrm{H}}$ during the rise (time interval C), but it seems that it is the combined effect of the variation in $N_{\mathrm{H}}$ and $\Gamma$ that drives the changes in most of the analyzed intervals. While we cannot firmly assess which spectral parameters are changing during observation 0154750601 due to the contamination from the flaring background, some other interesting HR variations were recorded during observation 0679810401 . We measured an increase in the absorption column density and hardening of the power law during the time intervals B and D that precede the peak of a faint flare of the source. Unfortunately, the observation ends before any data can be collected on the post-flare emission to verify a possible re-enhancement of the absorption column density with the decay of the X-ray flux.

- SAX J1818.6-1703: a progressive hardening has been measured in the only available observation during the rise to the peak of a relatively bright flare (time intervals A, B, C, D, and E). This is mostly due to the change in the power-law slope, with only a minor change in the absorption column density. However, we measured a sharp drop in the absorption column density slightly before the peak of the flare (time interval F). We also reported marginal evidence for a reenhancement of the local absorption following the peak of the flare (time interval G).

- IGRJ17354-3255: during observation 0693900201, the source displayed little (if any) spectral variability during the rise and drop from the two brightest X-ray flares, but showed a prominent HR increase between them that corresponds to a factor of $\gtrsim 3$ enhancement in the local absorption column density. In the other two observations, the source displays an increase in the absorption column density preceding the source X-ray flares (time intervals B and E in observation 0701230101, and $\mathrm{H}$ and $\mathrm{N}$ in observation 0701230701) and a decrease in the absorption column density during the brightest phases of the different flares (time intervals $\mathrm{C}$ and $\mathrm{F}$ in observation 0701230101 , and F, L, and $\mathrm{O}$ in observation 0701230701).

- IGR J16328-4732: observation 0679810201 shows that there seems to be little spectral variability during the decay from the first flare (time intervals A and B), while a significant increase in the local absorption column density precedes the second X-ray flare (time interval C). A drop in the absorption column density is recorded during the peaks of the flares, as shown by the results of the fits to the spectra in the time intervals $\mathrm{E}$ and $\mathrm{N}$. We also measured significant variations of the power-law slope, which generally becomes softer during the time intervals corresponding to relatively low flux states (B, C, D, and R). During observation 0679810301, the variations in the power-law slope were more pronounced and this made it difficult to measure the changes in the absorption column density. In particular, we could not find a clear indication of an increase in $N_{\mathrm{H}}$ before the rise to more prominent flares and a decrease during the brightest phases of these events (as we measured in observation 0679810201). Quite peculiar are the cases of the time intervals $\mathrm{C}, \mathrm{H}$, and $\mathrm{O}$, where a relatively high absorption column density is measured, although the corresponding value of the HR is low due to a significant steeping of the power-law slope. An interesting case is also that of the time interval D, which is characterized by the lowest absorption column density measured during observation and which is associated with the peak of a relatively faint $\mathrm{X}$-ray flare.

\section{Discussion}

The goal of the present study is to use XMM-Newton data to verify whether X-ray flares from the SFXTs can all be associated with the presence of intervening dense clumps from the supergiant star winds. Accurate measurements of the absorption column densities during the flares are mandatory to achieve this goal. For the analysis of all data, we adopted a technique that we verified in previous publications as a particularly powerful probe of the accretion physics in wind-fed X-ray binaries. In particular, we adaptively rebin the energy resolved light curves of all sources and use the correspondingly derived HR to drive the selection of the different time intervals for the spectral extraction.

The analysis of the flares reported in Sect. 2 and the corresponding results summarized in Sect. 3 show that, generally, an increase in the local absorption column density is recorded during the rise of these events; a drop follows when the source reaches the peak flux. In some cases a re-increase in the absorption column density after the flare is also detected. This phenomenology was already pointed out during our previous analysis of the bright flare from IGR J18410-0535 (Bozzo et al. 2011) and the outburst from IGR J17544-2619 (Bozzo et al. 2016a). Similar variations in the absorption column density are expected if the flares/outbursts are triggered by the presence of stellar wind clumps approaching the compact object. The drop in $N_{\mathrm{H}}$ close to the peak of the X-ray activity can most likely be ascribed to the photoionization of the clump medium. A re-increase in e $N_{\mathrm{H}}$ is expected after the flare, due to the recombination that is allowed by the decreasing X-ray flux. We also reported a new detection of an enhanced absorption event not connected with an X-ray flare during observation 0693900201 of the SFXT IGR J17354-3255. Few other similar cases were reported in the literature and associated with the passage of a clump along the observer line of sight to the source without being accreted. This is expected due to the double role of the clumps mentioned in Sect. 1.

In order to carry out a statistically meaningful analysis of the results given in Tables A.1-A.5, we also built for each source the distributions of the $N_{\mathrm{H}}$ as a function of the flux in Fig. 15. We plot for each observational time interval the measured absorption column density and flux normalized to the weighted average values (computed each time by excluding the reference time interval). We included the data from the previously published outburst of IGR J17544-2619 and the flare from IGR J184100535, as for these observations we had also exploited the HRresolved spectral analysis technique. We used the time interval selection presented in Fig. 6 of Bozzo et al. (2016a) and Fig. 7 of Bozzo et al. (2011) and re-fit the corresponding spectra with the PHABS*PEGPOW model in order to have consistent measurements of the spectral parameters and fluxes for the entire dataset. Using a normalized $N_{\mathrm{H}}$ allows us to carry out an easier comparison between the results obtained for the different sources and draw some preliminarily conclusion for the SFXT as a class.

Two main results emerge from Fig. 15. The first result is that there is no significant correlation between the dynamic range in the X-ray flux and the dynamic range in the absorption column density achieved by any of the sources. This means, for example, that sources achieving a broader dynamic range in the $\mathrm{X}$-ray flux do not necessarily display the largest swings in absorption column density. The widest dynamic range in $N_{\mathrm{H}}$ was measured during the bright flare from IGR J18410-0535 (a factor of $\sim 15$ ), which was also characterized by a dynamic range in the X-ray flux of a factor of 2000 . In the case of IGR J17544-2619, which underwent several bright flares and one outburst with a 

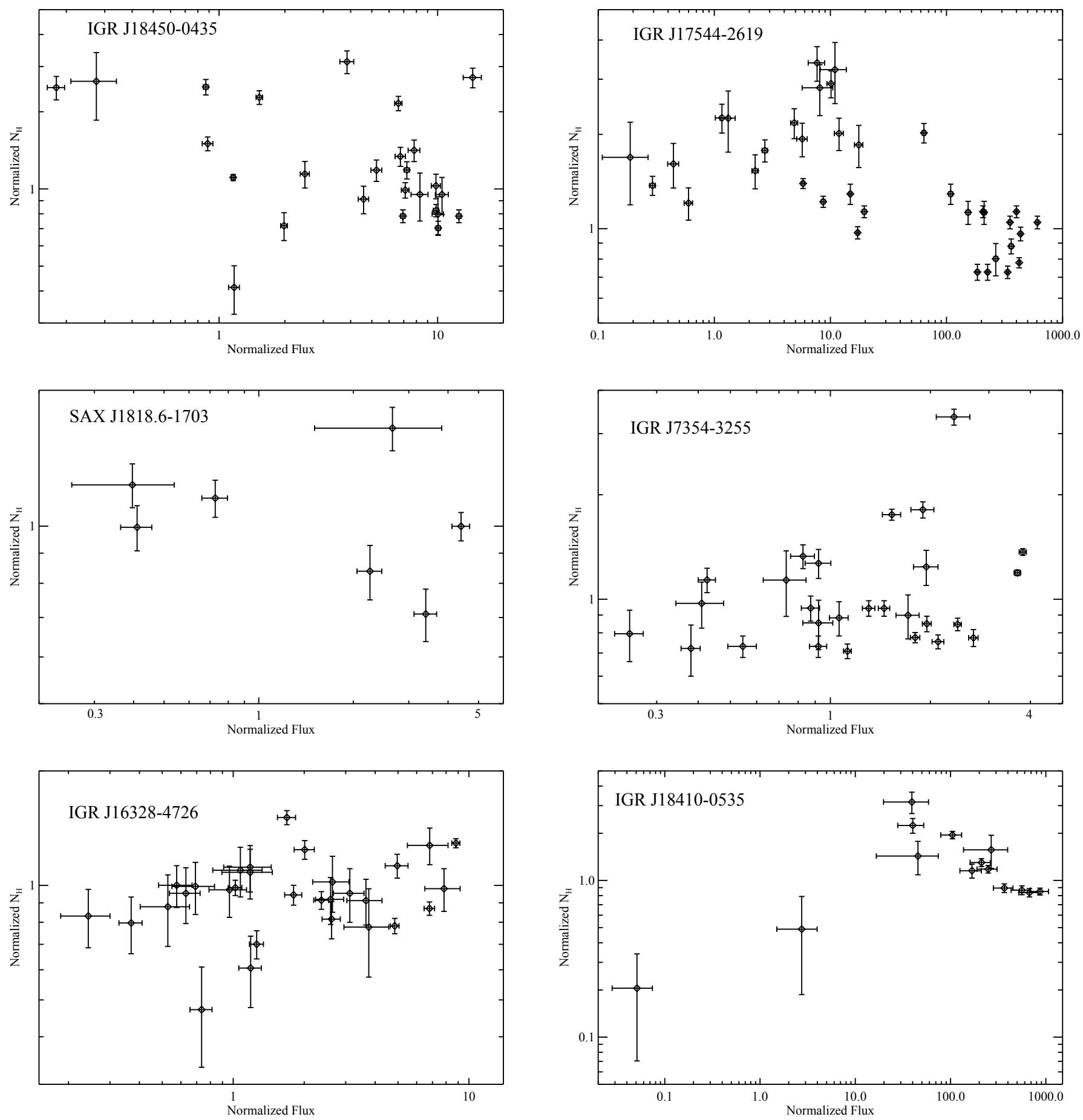

Fig. 15. Distributions of the normalized absorption column density as a function of the normalized flux (1-10 keV) measured from the HRresolved spectral analysis for the five sources in Sect. 2 (see text for further details). The previously published XMM-Newton data on the outburst of IGR J17544-2619 (Bozzo et al. 2016b) and the bright flare from IGR J18410-0535 (Bozzo et al. 2011) are also included. All uncertainties are at $1 \sigma$ confidence level.

total dynamic range in the X-ray flux of $\sim 3.5 \times 10^{3}$, the total dynamic range in the $N_{\mathrm{H}}$ was limited to a factor of $\sim 5$. A similar factor is measured in the case of IGR J18450-0435, which did not undergo an outburst but displayed several relatively bright flares (the total dynamic range in the X-ray flux was of $\lesssim 100$ ). More limited dynamic ranges in the absorption column density $(\lesssim 3)$ have been recorded in the cases of IGR J16328-4726 and IGR J7354-3255, even though these sources displayed a flux dynamic range similar to IGR J18450-0435 (the only exception is the case of IGR J7354-3255 if the highest recorded $N_{\mathrm{H}}$ corresponding to the passage of a clump in front of the X-ray source is taken into account). This is in agreement with the general idea that clumps cannot be the only ingredient to explain the SFXT extreme X-ray variability. If this were the case, the dynamic range in the X-ray flux should be directly proportional to the density contrast of the clump compared to the intra-clump medium and thus to the observed dynamic range of the absorption column density. Instead, we measure similar $N_{\mathrm{H}}$ swings in different 
Table 3. Weighted mean of the normalized absorption column density as a function of a chosen flux interval in the two SFXTs IGR J175442619 and IGR J18410-0535.

\begin{tabular}{|c|c|}
\hline Normalized flux range & Normalized $N_{\mathrm{H}}$ (uncertainty) \\
\hline \multicolumn{2}{|c|}{ IGR J17544-2619 } \\
\hline $0.1-1.0$ & $1.35(0.08)$ \\
\hline $1.0-20.0$ & $1.25(0.02)$ \\
\hline $20.0-1000.0$ & $0.90(0.01)$ \\
\hline \multicolumn{2}{|c|}{ IGR J18410-0535 } \\
\hline $0.01-10.0$ & $0.25(0.12)$ \\
\hline $10.0-100.0$ & $2.00(0.09)$ \\
\hline $100.0-1000.0$ & $0.95(0.02)$ \\
\hline
\end{tabular}

Notes. All uncertainties are given at $1 \sigma$ confidence level.

sources for X-ray dynamic ranges that differ by a factor of $\simeq 100$. The presence of mechanisms inhibiting the accretion of the stellar wind material in SFXTs can explain this discrepancy as they can boost the variability of these sources by switching between periods of quiescence and enhanced accretion (and vice versa) even when only moderate variations of the density of the stellar wind material take place in the surroundings of the compact object. This complication and the lack of a proper knowledge of the NS spin period and magnetic field intensity in the SFXTs prevent us fro efficiently using the measurements of the column density variations in the these sources to estimate the physical properties of the stellar clumps (see Sect. 1).

The second result that emerges from Fig. 15 is an indication that for the SFXT sources the absorption column densities measured at the highest fluxes are lower than those measured during the low and intermediate fluxes. Among all the sources in the present sample, IGR J17544-2619 and IGR J18410-0535 provide the clearest evidence for such phenomenon. This is not surprising because these are also the only two sources that have been observed by XMM-Newton in a sufficiently wide range of $\mathrm{X}$-ray flux to draw a more corroborated conclusion. To quantify this conclusion to the best of our current possibilities, we calculated the weighted mean and the standard deviation of the normalized $N_{\mathrm{H}}$ in different normalized flux intervals for the two sources. The results are provided in Table 3 . We note that for the SFXT sources a lower absorption column density at the highest fluxes compared to lower flux states is expected according to previous discussions in this section, as the largest increase in the absorption column density is measured during the rise of the flares/outbursts and a drop should occur above the threshold at which the clump material is significantly photoionized. Future XMM-Newton observations of the confirmed SFXTs during quiescence, flares, and outbursts will permit us to improve this analysis and carry out more quantitative statistical investigations of the entire class of sources.

\subsection{Classification of SFXT flares and outbursts}

Our poor understanding of the mechanisms inhibiting accretion in the SFXTs does not allow us to directly infer the properties of the stellar wind clumps; however, we can propose a preliminary classification of the enhanced accretion episodes from these sources to link the observations with the accretion physics and make prediction which could drive future investigations.
Based on our current theoretical understanding of the SFXT accretion physics and the evidence collected so far from the $\mathrm{X}$-ray observations, we propose the following four categories of flares/outbursts:

A: flares $\left(L_{\mathrm{X}} \simeq 10^{34}-10^{35} \mathrm{erg} \mathrm{s}^{-1}\right)$ that are generated by the impact of moderately dense structures. These dense structures are not able to fully overcome all the mechanisms inhibiting accretion, and thus accretion is only partly restored during the flare ${ }^{6}$. As these structures are not extremely massive/dense, the increase in the local absorption can be limited $\left(\Delta N_{\mathrm{H}} \lesssim 2-3\right)$ or even hidden within the uncertainty of the $N_{\mathrm{H}}$ in the direction of the source. If flares in category A are observed from a source, it cannot be excluded that brighter flares in the following category B can also occur, and outbursts in the categories $\mathrm{C}$ and $\mathrm{D}$ below as well.

B: flares $\left(L_{\mathrm{X}} \simeq 10^{34}-10^{35} \mathrm{erg} \mathrm{s}^{-1}\right)$ that are generated by the impact of sufficiently dense structures producing measurable increases in the local absorption $\left(\Delta N_{\mathrm{H}} \gg 3\right)$. If such flares are found in a system, it is expected that the mechanisms inhibiting accretion are more difficult to overcome as massive structures are needed to produce even moderately faint flares. Therefore, these sources should not show outbursts associated with modest or no increases in the absorption column density (category $\mathrm{C}$ below). It is still possible that flares belonging to category A might occur at lower luminosities.

C: outbursts $\left(L_{X} \simeq 10^{36}-10^{38} \mathrm{erg} \mathrm{s}^{-1}\right)$ that are generated by the impact of moderately massive structures. The limited increase in the absorption during these events $\left(\Delta N_{\mathrm{H}} \lesssim 2-3\right)$ indicates that the inhibiting mechanisms are particularly easy to overcome. We should thus expect frequent outbursts from these systems and also fainter flares which do not display at all the signature of a measurable increased X-ray extinction (as the partial inhibition of accretion is overcome even more easily; category A). Flares belonging to category B should not be expected from these systems.

D: outbursts $\left(L_{X} \simeq 10^{36}-10^{38} \mathrm{erg} \mathrm{s}^{-1}\right)$ that are generated by the impact of sufficiently dense structures to produce large increases in the absorption $\left(\Delta N_{\mathrm{H}} \gg 3\right)$. If the onset of an outburst requires the presence of these structures, these systems should show less frequent outbursts and a larger number of fainter flares (as for them it is, on average, more difficult to completely overcome the inhibition mechanisms). Flares from categories A and B can be expected; those in category B achieve higher luminosities.

In the XMM-Newton data available so far, we have found episodes in three of the above categories (A, B, and C), but outbursts giving rise to very large variations in the accretion column density ( $\gg 3$, case D) have not yet been observed (the only outburst observed by XMM-Newton is the one from IGR J175442619 and it is of type C). We can also use, however, all previously published results in the literature to perform a preliminary test of the proposed classification and of the applicability to different sources.

In the case of the SFXT prototype IGR J17544-2619, large increases in the absorption column density were not detected during the many outbursts observed with Swift/XRT (Romano et al. 2008a, 2011a, 2014), XMM-Newton (Bozzo et al. 2016a), and Suzaku (Rampy et al. 2009), or during the fainter flares analyzed here. The spectral variability seems more related to changes in the power-law photon index and so to the

6 It is generally believed that the inhibition of accretion is only completely removed during the outbursts (see Sect. 1). 
emission mechanism intrinsic to the source (see the exhaustive discussion in Bozzo et al. 2016b, and references therein), while the absorption column density does not change dramatically between quiescence and the very bright X-ray states (in 't Zand 2005). We should thus conclude that in the case of IGR J175442619 the mechanism(s) inhibiting the accretion can easily be overcome by moderate variations in the density of the accreting material (a factor of $\sim 2-3$ at the most) in order to produce both flares and outbursts (cases $\mathrm{A}$ and $\mathrm{C}$ of the classification). The prediction for IGR J17544-2619 is that during its future outbursts there will not be large absorption variations and that during its X-ray flares the variations should be even lower.

In the case of IGR J18450-0435, we recorded variations in $N_{\mathrm{H}}$ as large as a factor of $\sim 8$ during some bright flares (see Fig. 15) and we should thus conclude that overcoming the inhibition mechanisms for this source is not as easy as in the case of IGR J17544-2619. Large swings of the absorption column density would thus be expected during future flares and outbursts (cases B and D). In the sole outburst observed in the soft $\mathrm{X}$-ray domain from this source with Swift/XRT, no variations in $N_{\mathrm{H}}$ were reported (Romano et al. 2012a, 2013; Goossens et al. 2013). However, we note that some limitations in the observational capabilities of this instrument could hamper the searches for these variations within the required timescales and in the relevant phases of the event (see Sect. 4.1.1).

The analysis of the flares from SAX J1818.6-1703 reported in this paper did not reveal very large variations in the absorption column density, but the average $N_{\mathrm{H}}$ was as high as $3 \times 10^{23} \mathrm{~cm}^{-2}$. Similar high values of $N_{\mathrm{H}}$ have been measured by Swift/XRT during a number of previous outbursts/flares with swings down to a few times $10^{22} \mathrm{~cm}^{-2}$ (Sidoli et al. 2009b; Romano et al. 2009a,c; Kennea et al. 2014). Even though we do not have clear evidence of the absorption column density regularly increasing during the rise of these events and the properties of the source emission outside the outburst/flaring state are not known (two deep XMM-Newton observations carried out to this aim failed to detect the source in both occasions; see Bozzo et al. 2008a, 2012), we would first conclude that the mechanisms inhibiting accretion in SAX J1818.6-1703 cannot be overcome without the intervention of a substantially dense structure. We thus expect to detect with XMM-Newton much larger $N_{\mathrm{H}}$ variations during the rising phase of an outburst than during a flare (case D of the classification).

For IGR J17354-3255 our analysis did not reveal large HR variations corresponding to the rise and decay of the flares. At present, we might thus conclude that for this source overcoming the mechanisms inhibiting the accretion should not be very difficult as noticeable $\mathrm{X}$-ray flares are associated with modest increases in the absorption column density (a factor of $\lesssim 2$; case $\mathrm{A}$ of the classification). This does not preclude the existence of structures that have higher density in the stellar and that could pass in front of the compact object, causing its obscuration outside flares and outbursts (see also Sect. 1). In fact, we detected an increase in $N_{\mathrm{H}}$ between two flares that was most likely due to the passage of a clump along the observer's line of sight. More observations of this source in outburst with large area instruments are definitively needed in order to confirm this conclusion, as so far no outbursts from IGR J17354-3255 could be studied in the soft X-ray domain (see also Ducci et al. 2013, and references therein). Our prediction is that for this source no outbursts with large variations in $N_{\mathrm{H}}$ should be observed (case $\mathrm{C}$ of the classification).

In the case of IGR J16328-4726, the flares analyzed here show that there is usually some limited variability (a factor of $\sim 2-3$ ) in the absorption column density during these events (case A of the classification). It could thus be initially concluded that this source is similar to IGR J17354-3255. However, we note that the outburst that was followed up with BeppoSAX/MECS was characterized by large swings in the absorption column density, reaching factors as high as $\sim 7$ (Fiocchi et al. 2013). We can thus predict that an observation of the source with XMM-Newton during an outburst will most likely reveal large swings in the absorption column density (case D of the classification) and thus that this source is more similar to IGR J18450-0435. Brighter and less frequent flares associated with large variations of the $N_{\mathrm{H}}$ (category B) should also be observable.

In the case of IGR J18410-0535, only one single bright flare has been observed by XMM-Newton with variations in the absorption column density up to a factor of $\gtrsim 15$ (case $B$ of the classification). We should thus expect to observe only outbursts with large changes in $N_{\mathrm{H}}$ from this system. No other evidence of large increases in the absorption column density have been reported in the literature, but we note that the observations performed in the direction of the source with ASCA and SUZAKU were endowed with limited statistics to perform any meaningful HR-resolved spectral analysis, and Swift/XRT observed the source thousand of seconds after the onset of all three detected outbursts (Bamba et al. 2001; de Pasquale et al. 2010; Romano et al. 2010, 2011b, 2012c,b; Mangano et al. 2011; Kawabata et al. 2012).

There are a number of SFXTs which have not been discussed in this paper due to the lack of reasonable $\mathrm{S} / \mathrm{N}$ data, which makes it difficult to understand whether or not they follow the classification and predictions proposed above. XTEJ1739-302 and IGR J16479-4514 have been observed in outbursts many times with Swift/XRT and thus several measurements of the local absorption column density have been reported in the literature. For XTEJ1739-302 an enhanced $N_{\mathrm{H}}$ at the on-set of the outbursts compared to quiescent states has clearly been detected in several cases (Sidoli et al. 2008, 2009a,c), even though the usually fragmented Swift/XRT light curves do not allow an in depth analysis of the events to be carried out. The only observations of the source available with XMM-Newton caught the system in quiescence and the flares detected are too faint to investigate the presence of any spectral variability (see Bozzo et al. 2010; Bodaghee et al. 2011). Large swings of the absorption column density have been detected during the outbursts of the SFXT IGR J08408-4503, which seems to display on average a much harder emission during the active X-ray states than during quiescence (Romano et al. 2009b; Bozzo et al. 2010; Sidoli et al. 2010; Mangano et al. 2013). At present, we would thus first conclude that XTEJ1739-302 and IGR J08408-4503 are similar to IGR J18450-0435, SAX J1818.6-1703, and IGR J163284726 with regard to the flares/outbursts classification and the accretion inhibition mechanisms, but this conclusion needs to be corroborated by additional X-ray observations during flaring or outburst states. We conclude the opposite for IGR J164794514 , which displayed evidence of changes in the local absorption column density especially during the eclipses (Sidoli et al. 2008; Bozzo et al. 2008c; Sidoli et al. 2013), but most likely not during the rise to the few outbursts observed with Swift/XRT (Romano et al. 2009d, 2008b; La Parola et al. 2009).

\subsubsection{Caveats to the flares/outbursts classification}

Even though it seems that all the SFXTs that could be carefully checked so far qualitatively follow the proposed classification of 
flares/outbursts and the predictions presented in Sect. 4.1, there are two caveats that need to be taken into account:

- Impact of geometrical effects. The values of the absorption column density measured during the rising or decaying stages of an SFXT flare or outburst are likely affected by the projection effects related to the viewing angle between the observer line of sight and the direction from which the clump approaches the compact object. These effects have been discussed in theoretical and observational papers (Walter \& Zurita Heras 2007; Ducci et al. 2010; Bozzo et al. 2011). They are known to give rise to large uncertainties in the estimate of the clump size from the duration and intensity of the X-ray flare or outburst. In this respect, when no variation in the absorption column density is observed, it is difficult to conclude whether this is due to the lack of dense enough structures gathering around the NS or to a projection effect that might limit our ability to reveal those structures. Clearly, in all those cases in which a significant $N_{\mathrm{H}}$ increase is measured, any possible geometrical effect would only enhance it further.

- Observability of the variation of the absorption column density during the relevant stages of the flare/outburst. XMMNewton results have shown that the absorption column density might only increase during the rising or the final phases of the flare/outburst, while it can be comparable to the value measured during quiescence in other phases of the same event. As most of the SFXT flares/outbursts were observed with Swift/XRT between a hundred and a thousand seconds after the onset of the event, it is possible that in most of the cases we missed the large swings in the absorption column density. Uninterrupted observations of many more flares and outbursts with XMM-Newton are thus needed in order to further test our proposed classification and predictions.

\subsection{Stellar wind clumps and corotating interaction regions}

Since the early discovery of the SFXTs, it has always been assumed that the density variations in the material around the compact object triggering the onset of flares/outbursts were associated with the presence of clumps. This assumption followed especially from the need of having sufficiently dense and compact structures to trigger the SFXT fast X-ray flares within the required short timescales (a few hundred seconds) and explain the entire observed dynamic range in the SFXT X-ray luminosity only through the density contrast between the clump and the intra-clump medium. This need has been largely alleviated by the observation that all SFXTs are largely subluminous than classical systems, and that some mechanisms should be at work to inhibit accretion in these systems. These mechanisms act to boost the dynamical range in the X-ray luminosity achievable by the SFXTs, which in turn is no longer bound to the sole density ratio between the structure being accreted onto the NS and the surrounding stellar wind material.

Studies of isolated supergiants have shown that, in addition to clumps, at least one other kind of large-scale structure can be formed within the wind of an OB supergiant star, i.e., the corotating interaction regions (CIRs; Mullan 1984). The presence and properties of these structures are mainly inferred from the detection of discrete absorption components (DACs) in the UV spectra of many OB supergiants (Bates \& Gilheany 1990; Howarth 1992), and used in several cases to interpret the X-ray variability of these objects (Oskinova et al. 2001; Massa et al. 2014; Nazé et al. 2013). These DACs are revealed in the form of optical depth enhancements in the absorption troughs of unsaturated UV P Cygni profiles and were directly observed to accelerate towards the blue wing of the line profile, becoming narrower as they approach the limiting value of the stellar wind asymptotic velocity. The timescales on which these features move along the line profile is a few days, and it thus seems more plausible that they are connected with the star rotational period rather than with the intrinsic acceleration of the stellar wind (occurring on much short timescales if a relatively standard terminal velocity of few thousands $\mathrm{km} \mathrm{s}^{-1}$ is assumed for a typical OB supergiant). The widely agreed hypothesis is that DACs are the outcomes of slowly evolving velocity and/or density perturbations along the main stellar wind stream, originating from a substantial fraction of the stellar surface (see, e.g., Prinja \& Smith 1992; Prinja 1994, and references therein). Hydrodynamical simulations have shown that photospheric disturbances on the stellar surface can generate streams of enhanced density along the profile of an unperturbed wind, with a significant velocity drop at the edge of these streams where they interact with the unperturbed wind (Feldmeier \& Shlosman 2000, 2002). Even though the details on the formation and evolution of these structures are still under study (Hamann et al. 2001; Lobel \& Blomme 2008) and the overall picture of large-scale structures in the winds of OB supergiant could be complicated by the later discovery of the so-called rotational modulations (see, e.g., Lobel 2013, and references therein), it seems that the typical density and/or velocity contrasts of these structures with respect to the surrounding wind could be as large as a factor of $\sim 3$ (Cranmer \& Owocki 1996). The presence of these large and mildly dense structures could thus provide the required changes in the physical properties of the accretion medium that in turn can overcome the accretion inhibition mechanisms and trigger those SFXT flares/outbursts in which large variations in the local accretion column densities are not measured (cases $\mathrm{A}$ and $\mathrm{C}$ of our classification). It is not clear at present whether CIRs or other similar large-scale structures could survive in the heavily clumped wind of an $\mathrm{OB}$ supergiant (the instability usually adopted to generate clumps in the hydrodynamical simulations of their winds is not included in the simulations developed to produce the CIRs), but we propose here that many of the enhanced X-ray emission episodes that we detect from SFXTs could be associated with these structures rather than with more confined and dense clumps. As the CIRs are supposed to be somehow connected with the rotational velocity of the star, there is the interesting possibility that they could induce a periodical modulation of the X-ray luminosity in classical SgXBs and in SFXTs on much longer timescales than those of the flares/outbursts or the binary revolution (e.g., giving rise to the observed super-orbital modulation; see Corbet \& Krimm 2013). The analysis of this possibility and a full exploitation of the expected effect of CIRs on the X-ray emission of SFXTs and classical $\mathrm{SgXBs}$ is beyond the scope of the current paper and will be reported in a forthcoming publication (Bozzo et al., in prep.).

Acknowledgements. We thank the anonymous referee for the constructive comments which helped to improve the paper. This publication was motivated by a team meeting sponsored by the International Space Science Institute in Bern, Switzerland. E.B. and L.O. thank ISSI for the financial support during their stay in Bern. E.B. is grateful for the hospitality of the New York University Abu Dhabi during part of this work. P.R. and E.B. acknowledge the financial contribution from the agreement ASI-INAF I/037/12/0. P.R. acknowledges grant ASIINAF I/004/11/0. L.O. acknowledges support by the DLR grant 50 OR 1302 and partial support by the Russian Government Program of Competitive Growth of Kazan Federal University. 


\section{References}

Bamba, A., Yokogawa, J., Ueno, M., Koyama, K., \& Yamauchi, S. 2001, PASJ, 53,1179

Bates, B., \& Gilheany, S. 1990, MNRAS, 243, 320

Bodaghee, A., Tomsick, J. A., Rodriguez, J., et al. 2011, ApJ, 727, 59

Boon, C. M., Bird, A. J., Hill, A. B., et al. 2016, MNRAS, 456, 4111

Bozzo, E., Campana, S., Stella, L., et al. 2008a, ATel, 1493

Bozzo, E., Falanga, M., \& Stella, L. 2008b, ApJ, 683, 1031

Bozzo, E., Stella, L., Israel, G., Falanga, M., \& Campana, S. 2008c, MNRAS, 391, L108

Bozzo, E., Stella, L., Ferrigno, C., et al. 2010, A\&A, 519, A6

Bozzo, E., Giunta, A., Cusumano, G., et al. 2011, A\&A, 531, A130

Bozzo, E., Pavan, L., Ferrigno, C., et al. 2012, A\&A, 544, A118

Bozzo, E., Romano, P., Ferrigno, C., Esposito, P., \& Mangano, V. 2013, Adv Space Res., 51, 1593

Bozzo, E., Romano, P., Ducci, L., Bernardini, F., \& Falanga, M. 2015, Adv. Space Res., 55, 1255

Bozzo, E., Bhalerao, V., Pradhan, P., et al. 2016a, A\&A, 596, A16

Bozzo, E., Oskinova, L., Feldmeier, A., \& Falanga, M. 2016b, A\&A, 589, A102

Corbet, R. H. D., \& Krimm, H. A. 2013, ApJ, 778, 45

Courvoisier, T. J.-L., Walter, R., Beckmann, V., et al. 2003, A\&A, 411, L53

Cranmer, S. R., \& Owocki, S. P. 1996, ApJ, 462, 469

Dame, T. M., Hartmann, D., \& Thaddeus, P. 2001, ApJ, 547, 792

de Pasquale, M., Barthelmy, S. D., Baumgartner, W. H., et al. 2010, ATel, 2661

Dickey, J. M., \& Lockman, F. J. 1990, ARA\&A, 28, 215

Drave, S. P., Bird, A. J., Sidoli, L., et al. 2013, MNRAS, 433, 528

Drave, S. P., Bird, A. J., Sidoli, L., et al. 2014, MNRAS, 439, 2175

Ducci, L., Sidoli, L., \& Paizis, A. 2010, MNRAS, 408, 1540

Ducci, L., Romano, P., Esposito, P., et al. 2013, A\&A, 556, A72

Feldmeier, A., \& Shlosman, I. 2000, ApJ, 532, L125

Feldmeier, A., \& Shlosman, I. 2002, ApJ, 564, 385

Fiocchi, M., Bazzano, A., Bird, A. J., et al. 2013, ApJ, 762, 19

Fiocchi, M., Bazzano, A., Natalucci, L., et al. 2016, ApJ, 829, 125

Giménez-García, A., Shenar, T., Torrejón, J. M., et al. 2016, A\&A, 591, A26

González-Riestra, R., Oosterbroek, T., Kuulkers, E., Orr, A., \& Parmar, A. N. 2004, A\&A, 420, 589

Goossens, M. E., Bird, A. J., Drave, S. P., et al. 2013, MNRAS, 434, 2182

Grebenev, S. A., \& Sunyaev, R. A. 2007, Astron. Lett., 33, 149

Grinberg, V., Leutenegger, M. A., Hell, N., et al. 2015, A\&A, 576, A117

Hamann, W.-R., Brown, J. C., Feldmeier, A., \& Oskinova, L. M. 2001, A\&A 378, 946

Howarth, I. D. 1992, in Nonisotropic and Variable Outflows from Stars, eds.

L. Drissen, C. Leitherer, \& A. Nota, ASP Conf. Ser., 22, 155

in 't Zand, J. J. M. 2005, A\&A, 441, L1

Jansen, F., Lumb, D., Altieri, B., et al. 2001, A\&A, 365, L1

Kawabata, K., Nobukawa, M., Tsuru, T. G., \& Koyama, K. 2012, PASJ, 64, 99

Kennea, J. A., Krimm, H. A., Romano, P., et al. 2014, ATel, 5980

La Parola, V., Romano, P., Sidoli, L., et al. 2009, ATel, 1929

Lobel, A. 2013, in Massive Stars: From alpha to Omega, 43

Lobel, A., \& Blomme, R. 2008, ApJ, 678, 408
Lutovinov, A. A., Revnivtsev, M. G., Tsygankov, S. S., \& Krivonos, R. A. 2013, MNRAS, 431, 327

Mangano, V., Barthelmy, S. D., Romano, P., et al. 2011, ATel, 3453

Mangano, V., Romano, P., Ceccobello, C., \& Farinelli, R. 2013, Mem. Soc. Astron. It., 84, 604

Martínez-Núñez, S., Kretschmar, P., Bozzo, E., et al. 2017, Space Sci. Rev., 212, 59

Massa, D., Oskinova, L., Fullerton, A. W., et al. 2014, MNRAS, 441, 2173

Mullan, D. J. 1984, ApJ, 283, 303

Nazé, Y., Oskinova, L. M., \& Gosset, E. 2013, ApJ, 763, 143

Negueruela, I. 2010, in High Energy Phenomena in Massive Stars, eds. J. Martí, P. L. Luque-Escamilla, \& J. A. Combi, ASP Conf. Ser., 422, 57

Oskinova, L. M., Clarke, D., \& Pollock, A. M. T. 2001, A\&A, 378, L21

Prinja, R. K. 1994, in Pulsation; Rotation; and Mass Loss in Early-Type Stars, eds. L. A. Balona, H. F. Henrichs, \& J. M. Le Contel, IAU Symp., 162, 507

Prinja, R. K., \& Smith, L. J. 1992, A\&A, 266, 377

Rampy, R. A., Smith, D. M., \& Negueruela, I. 2009, ApJ, 707, 243

Romano, P., Cusumano, G., Sidoli, L., et al. 2008a, ATel, 1697

Romano, P., Sidoli, L., Mangano, V., et al. 2008b, ApJ, 680, L137

Romano, P., Barthelmy, S., Sidoli, L., et al. 2009a, ATel, 2191

Romano, P., Sidoli, L., Cusumano, G., et al. 2009b, MNRAS, 392, 45

Romano, P., Sidoli, L., Krimm, H. A., et al. 2009c, ATel, 2044

Romano, P., Sidoli, L., Mangano, V., et al. 2009d, ATel, 1920

Romano, P., Cusumano, G., Baumgartner, W. H., et al. 2010, ATel, 2662

Romano, P., La Parola, V., Vercellone, S., et al. 2011a, MNRAS, 410, 1825

Romano, P., Mangano, V., Cusumano, G., et al. 2011b, MNRAS, 412, L30

Romano, P., Barthelmy, S. D., Chester, M. M., et al. 2012a, ATel, 4095

Romano, P., Barthelmy, S. D., Kennea, J. A., et al. 2012b, ATel, 4176

Romano, P., Krimm, H., Sbarufatti, B., et al. 2012c, ATel, 4276

Romano, P., Mangano, V., Ducci, L., et al. 2013, Adv. Space Res., 52, 1593

Romano, P., Krimm, H. A., Palmer, D. M., et al. 2014, A\&A, 562, A2

Romano, P., Bozzo, E., Mangano, V., et al. 2015, A\&A, 576, L4

Shakura, N., Postnov, K., Kochetkova, A., \& Hjalmarsdotter, L. 2012, MNRAS, 420, 216

Shakura, N., Postnov, k., Sidoli, L., \& Paizis, A. 2014, MNRAS, 442, 2325

Sidoli, L., Romano, P., Mangano, V., et al. 2008, ApJ, 687, 1230

Sidoli, L., Romano, P., Ducci, L., et al. 2009a, MNRAS, 397, 1528

Sidoli, L., Romano, P., Esposito, P., et al. 2009b, MNRAS, 400, 258

Sidoli, L., Romano, P., Mangano, V., et al. 2009c, ApJ, 690, 120

Sidoli, L., Esposito, P., \& Ducci, L. 2010, MNRAS, 409, 611

Sidoli, L., Esposito, P., Sguera, V., et al. 2013, MNRAS, 429, 2763

Strüder, L., Briel, U., Dennerl, K., et al. 2001, A\&A, 365, L18

Tomsick, J. A., Chaty, S., Rodriguez, J., et al. 2009, ApJ, 694, 344

Torrejón, J. M., Schulz, N. S., Nowak, M. A., \& Kallman, T. R. 2010, ApJ, 715 947

Turner, M. J. L., Abbey, A., Arnaud, M., et al. 2001, A\&A, 365, L27

Walter, R., Lutovinov, A. A., Bozzo, E., \& Tsygankov, S. S. 2015, A\&ARv, 23, 2

Walter, R., \& Zurita Heras, J. 2007, A\&A, 476, 335

Zurita Heras, J. A., \& Walter, R. 2009, A\&A, 494, 1013 
E. Bozzo et al.: The accretion environment of supergiant fast X-ray transients probed with XMM-Newton

\section{Appendix A: Detailed results of the fits to the HR-resolved spectral analysis}

We present all the tables containing the detailed results of the HR-resolved spectral analysis performed for all sources and all observations.

Table A.1. Results of the fits to the A-Z spectra of IGR J18450-0435 in observation 0306170401 (see Fig. 1, top) and the A-H spectra of the same source in observation 0728370801 (see Fig. 3, second plot from the top).

\begin{tabular}{|c|c|c|c|c|c|c|c|}
\hline S. & $N_{\mathrm{H}}$ & $\Gamma$ & Flux & $C_{\mathrm{MOS} 1}$ & $C_{\mathrm{MOS} 2}$ & $\begin{array}{l}\chi_{\text {red }}^{2} / \\
\text { d.o.f. }\end{array}$ & $\begin{array}{l}\text { Exp. } \\
\text { (ks) }\end{array}$ \\
\hline \multicolumn{8}{|c|}{ Observation 0306170401} \\
\hline $\mathrm{A}^{a}$ & $3.0 \pm 0.6$ & $1.0 \pm 0.2$ & $4.4_{-0.3}^{+0.4}$ & $1.1 \pm 0.2$ & $1.1 \pm 0.1$ & $1.40 / 69$ & 0.32 \\
\hline B & $3.5 \pm 0.5$ & $1.4 \pm 0.2$ & $12.1_{-1.0}^{+1.2}$ & $1.1 \pm 0.1$ & $1.0 \pm 0.1$ & $1.19 / 81$ & 0.17 \\
\hline $\mathrm{C}$ & $2.6 \pm 0.3$ & $1.3 \pm 0.1$ & $12.7_{-0.8}^{+0.9}$ & $1.2 \pm 0.2$ & $1.2 \pm 0.1$ & $0.96 / 82$ & 0.25 \\
\hline $\mathrm{D}$ & $3.1 \pm 0.4$ & $1.4 \pm 0.1$ & $12.8_{-0.9}^{+1.0}$ & $1.2 \pm 0.1$ & $0.9 \pm 0.1$ & $1.18 / 116$ & 0.27 \\
\hline $\mathrm{E}$ & $1.1 \pm 0.4$ & $0.6 \pm 0.2$ & $2.1 \pm 0.2$ & $1.0 \pm 0.2$ & $0.9 \pm 0.1$ & $1.20 / 61$ & 0.60 \\
\hline $\mathrm{F}$ & $1.9 \pm 0.4$ & $0.9 \pm 0.2$ & $3.5 \pm 0.2$ & $1.0 \pm 0.2$ & $0.9 \pm 0.1$ & $0.79 / 64$ & 0.35 \\
\hline G & $3.1 \pm 0.5$ & $1.4 \pm 0.2$ & $9.4_{-0.8}^{+1.0}$ & $1.3 \pm 0.2$ & $1.4 \pm 0.2$ & $0.90 / 52$ & 0.20 \\
\hline $\mathrm{H}$ & $2.5 \pm 0.7$ & $1.3 \pm 0.2$ & $18.8_{-2.0}^{+2.3}$ & $1.2 \pm 0.2$ & $1.1 \pm 0.2$ & $0.90 / 37$ & 0.09 \\
\hline $\mathrm{L}^{a}$ & $2.5 \pm 0.9$ & $1.4 \pm 0.3$ & $14.9_{-2.0}^{+2.5}$ & $1.3 \pm 0.2$ & $1.1 \pm 0.2$ & $1.47 / 20$ & 0.09 \\
\hline M & $2.2 \pm 0.2$ & $1.3 \pm 0.1$ & $17.5 \pm 0.8$ & $0.97 \pm 0.06$ & $0.93 \pm 0.06$ & $1.10 / 176$ & 0.35 \\
\hline $\mathrm{N}^{a}$ & $2.4 \pm 0.5$ & $1.1 \pm 0.2$ & $8.2_{-0.7}^{+0.8}$ & $1.1 \pm 0.1$ & $0.9 \pm 0.1$ & $1.23 / 58$ & 0.26 \\
\hline $\mathrm{O}$ & $3.7 \pm 0.6$ & $1.5 \pm 0.2$ & $14.0_{-1.3}^{+1.6}$ & $1.3 \pm 0.2$ & $1.3 \pm 0.2$ & $0.97 / 58$ & 0.15 \\
\hline $\mathrm{P}$ & $2.7 \pm 0.5$ & $1.3 \pm 0.2$ & $17.6_{-1.3}^{+1.5}$ & $1.1 \pm 0.2$ & $1.2 \pm 0.2$ & $0.98 / 74$ & 0.18 \\
\hline $\mathrm{Q}^{a}$ & $2.1 \pm 0.6$ & $1.1 \pm 0.2$ & $18.0_{-1.7}^{+1.9}$ & $1.1 \pm 0.1$ & $1.0 \pm 0.1$ & $1.26 / 42$ & 0.10 \\
\hline $\mathrm{R}$ & $2.1 \pm 0.2$ & $1.14 \pm 0.07$ & $22.2 \pm 0.8$ & $1.15 \pm 0.06$ & $1.0 \pm 0.06$ & $1.18 / 219$ & 0.62 \\
\hline S & $1.9 \pm 0.2$ & $1.1 \pm 0.1$ & $17.8_{-0.6}^{+0.7}$ & $1.1 \pm 0.1$ & $1.0 \pm 0.1$ & $1.15 / 166$ & 0.33 \\
\hline $\mathrm{T}$ & $2.1 \pm 0.2$ & $1.1 \pm 0.1$ & $12.2_{-0.4}^{+0.5}$ & $1.1 \pm 0.1$ & $1.1 \pm 0.1$ & $1.11 / 208$ & 0.62 \\
\hline $\mathrm{Z}^{b}$ & $2.8 \pm 0.1$ & $1.14 \pm 0.04$ & $1.94 \pm 0.04$ & $1.05 \pm 0.03$ & $1.00 \pm 0.03$ & $1.50 / 357$ & 11.72 \\
\hline \multicolumn{8}{|c|}{ Observation 0728370801} \\
\hline $\mathrm{A}$ & $6.5_{-1.1}^{+1.2}$ & $1.6 \pm 0.3$ & $0.39_{-0.05}^{+0.07}$ & $1.1 \pm 0.1$ & $1.0 \pm 0.1$ & $1.01 / 78$ & 7.1 \\
\hline B & $5.9 \pm 0.6$ & $1.1 \pm 0.1$ & $2.7_{-0.1}^{+0.2}$ & $0.98 \pm 0.07$ & $0.97 \pm 0.07$ & $1.08 / 170$ & 2.4 \\
\hline $\mathrm{C}$ & $8.2_{-1.3}^{+1.5}$ & $1.2 \pm 0.2$ & $6.9_{-0.7}^{+1.0}$ & $1.0 \pm 0.1$ & $1.1 \pm 0.1$ & $0.86 / 80$ & 0.5 \\
\hline $\mathrm{D}$ & $5.6 \pm 0.6$ & $1.1 \pm 0.1$ & $11.8_{-0.7}^{+0.8}$ & $1.09 \pm 0.08$ & $0.98 \pm 0.08$ & $0.97 / 131$ & 0.5 \\
\hline $\mathrm{E}$ & $7.1_{-1.0}^{+1.1}$ & $1.8 \pm 0.3$ & $26.0_{-3.5}^{+4.9}$ & $1.1 \pm 0.1$ & $1.0 \pm 0.1$ & $1.08 / 78$ & 0.2 \\
\hline $\mathrm{F}$ & $6.5_{-0.7}^{+0.8}$ & $1.1 \pm 0.2$ & $1.60_{-0.06}^{+0.07}$ & $1.08 \pm 0.08$ & $1.07 \pm 0.08$ & $1.14 / 161$ & 3.9 \\
\hline G & $3.9 \pm 0.4$ & $0.8 \pm 0.1$ & $1.6_{-0.1}^{+0.2}$ & $0.92 \pm 0.05$ & $0.92 \pm 0.05$ & $1.08 / 215$ & 4.9 \\
\hline$\underline{\mathrm{H}}$ & $6.9_{-2.8}^{+4.1}$ & $1.2_{-0.6}^{+0.7}$ & $0.5_{-0.1}^{+0.3}$ & $1.2 \pm 0.2$ & $1.0 \pm 0.2$ & $0.46 / 17$ & 1.5 \\
\hline
\end{tabular}

Notes. The absorption column density is measured in units of $10^{22} \mathrm{~cm}^{-2}$, while the $1-10 \mathrm{keV}$ flux is given in units of $10^{-11} \mathrm{erg} \mathrm{cm}^{-2} \mathrm{~s}^{-1}$. ${ }^{(a)} \mathrm{In}$ these cases we verified that the slightly high $\chi_{\text {red }}^{2}$ values were only due to the noise in reduced statistics spectra rather than evident trends in the residuals (which could have suggested that a more refined model was needed to fit the data). ${ }^{(b)}$ This spectrum could not be well fit by using a simple absorbed power-law model. See Sect. 2.1 for details.
Table A.2. Same as Table A.1, but for the case of IGR J17544-2619 observation 0148090501 (Fig. 5), 0154750601 (Fig. 6), and 0679810401 (Fig. 7).

\begin{tabular}{|c|c|c|c|c|c|c|c|}
\hline S. & ${ }^{a}$ & $\Gamma$ & Flux & 1 & $\$ 2$ & $\begin{array}{l}\chi_{\text {red }}^{2} / \\
\text { d.o.f. }\end{array}$ & $\begin{array}{l}x p \\
\text { ks) }\end{array}$ \\
\hline \multicolumn{8}{|c|}{ Observation 0148090501} \\
\hline A & $2.1_{-0.8}^{1.3}$ & $2.6_{-0.6}^{+0.8}$ & $0.09_{-0.02}^{+0.11}$ & $1.2 \pm 0.4$ & $1.3 \pm 0.6$ & $0.60 / 8$ & 2.05 \\
\hline B & $2.8_{-0.9}^{+1.2}$ & $1.7 \pm 0.5$ & $0.6_{-0.1}^{+0.2}$ & $1.1 \pm 0.3$ & $1.1 \pm 0.4$ & $1.05 / 51$ & 0.42 \\
\hline $\mathrm{C}$ & $3.6 \pm 0.6$ & $1.8 \pm 0.3$ & $4.6_{-0.5}^{+0.7}$ & $1.0 \pm 0.1$ & $1.4 \pm 0.2$ & $1.02 / 62$ & 0.32 \\
\hline $\mathrm{D}$ & $4.2_{-0.8}^{+1.0}$ & $2.2 \pm 0.4$ & $3.5_{-0.7}^{+1.2}$ & $1.0 \pm 0.2$ & $1.2 \pm 0.3$ & $0.97 / 30$ & 0.27 \\
\hline $\mathrm{E}$ & $3.5 \pm 1.1$ & $2.4 \pm 0.7$ & $3.7_{-1.1}^{+2.6}$ & $1.2 \pm 0.3$ & $1.4 \pm 0.5$ & $0.60 / 10$ & 0.10 \\
\hline $\mathrm{F}$ & $2.7 \pm 0.5$ & $1.8 \pm 0.2$ & $2.2_{-0}^{+0}$ & $1.1 \pm 0.1$ & $1.5 \pm 0.3$ & $0.95 / 69$ & 0.60 \\
\hline$G$ & $2.8 \pm 0.5$ & $2.4 \pm 0.3$ & $0.53_{-0.09}^{+0.13}$ & $1.2 \pm 0.2$ & 3 & $14 / 53$ & 1.72 \\
\hline \multicolumn{8}{|c|}{ Observation 0154750601} \\
\hline$\overline{\mathrm{A}^{a}}$ & $2.5 \pm 0.5$ & $1.8 \pm 0.3$ & $5.4_{-0.7}^{+0.9}$ & 1.0 & $1.0 \pm 0.1$ & $1.11 / 52$ & 0.32 \\
\hline $\mathrm{B}^{a}$ & $2.3_{-0.5}^{+0.7}$ & $1.4 \pm 0.3$ & $8.0_{-1.0}^{+1.2}$ & 1.0 & $1.0 \pm 0.1$ & $0.95 / 33$ & 0.15 \\
\hline $\mathrm{C}^{a}$ & $4.0 \pm 1.5$ & $2.3 \pm 0.6$ & $5.0_{-1.4}^{+2.9}$ & 1.0 & $0 \pm 0.2$ & $50 / 19$ & 0.20 \\
\hline 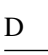 & $2.4_{-0.4}^{0.6}$ & $2.0 \pm 0.5$ & $2.6_{-0.4}^{+0.5}$ & $0.9 \pm 0.1$ & $9 \pm 0.1$ & $0.82 / 30$ & 0.30 \\
\hline \multicolumn{8}{|c|}{ Observation 0679810401} \\
\hline A & $2.0_{-0.5}^{0.6}$ & $2.1 \pm 0.3$ & $0.22 \pm 0.04$ & $0.9 \pm 0.2$ & $0.9 \pm 0.2$ & $0.94 / 27$ & 1.40 \\
\hline B & $1.7 \pm 0.2$ & $2.0 \pm 0.1$ & $0.22 \pm 0.02$ & $0.97 \pm 0.09$ & $0.99 \pm 0.09$ & $1.01 / 130$ & 8.07 \\
\hline $\mathrm{C}$ & $1.5 \pm 0.3$ & $1.8 \pm 0.3$ & $0.29 \pm 0.04$ & $0.9 \pm 0.1$ & $1.0 \pm 0.1$ & $1.17 / 33$ & 1.15 \\
\hline $\mathrm{D}$ & $2.2 \pm 0.3$ & $1.8 \pm 0.2$ & $1.2 \pm 0.1$ & $1.0 \pm 0.1$ & $1.1 \pm 0.1$ & $0.92 / 80$ & 0.87 \\
\hline $\mathrm{E}$ & $1.9 \pm 0.4$ & $1.7 \pm 0.2$ & $1.0 \pm 0.1$ & $1.1 \pm 0.2$ & $1.1 \pm 0.2$ & $0.87 / 45$ & 0.56 \\
\hline
\end{tabular}

Notes. ${ }^{(a)}$ For these spectra no EPIC-pn data were available, and thus the normalization constant of the MOS1 was fixed to unity and only that of the MOS2 was left free to vary in the fit.

Table A.3. Same as Table A.1, but for the case of SAX J1818.6-1703 observation 0679810501.

\begin{tabular}{llllllll}
\hline \hline S. $N_{\mathrm{H}}$ & $\Gamma$ & Flux & $C_{\text {MOS1 }}$ & $C_{\text {MOS2 }}$ & $\begin{array}{l}\chi_{\text {red }}^{2} / \\
\text { d.o.f. }\end{array}$ & $\begin{array}{l}\text { Exp. } \\
(\mathrm{ks})\end{array}$ \\
\hline A & $32.9 \pm 4.5$ & $2.8 \pm 0.4$ & $3.3_{-1.3}^{+2.7}$ & $0.97 \pm 0.1$ & $1.0 \pm 0.1$ & $1.05 / 85$ & 3.8 \\
B & $40.6 \pm 5.5$ & $3.0 \pm 0.5$ & $21.2_{-9.5}^{+21.7}$ & $1.0 \pm 0.1$ & $1.0 \pm 0.1$ & $0.94 / 69$ & 0.9 \\
C & $28.3 \pm 4.0$ & $0.8 \pm 0.3$ & $4.4_{-0.7}^{+0.9}$ & $1.0 \pm 0.1$ & $1.0 \pm 0.1$ & $0.86 / 93$ & 1.2 \\
D & $31.2 \pm 3.5$ & $0.8 \pm 0.2$ & $6.4_{-0.8}^{+1.0}$ & $0.93 \pm 0.08$ & $0.97 \pm 0.09$ & $1.11 / 123$ & 1.1 \\
E & $24.2 \pm 4.2$ & $0.0 \pm 0.3$ & $16.9_{-2.0}^{+2.6}$ & - & - & $0.84 / 66$ & 0.4 \\
F & $21.0 \pm 3.5$ & $0.0 \pm 0.2$ & $25.3_{-2.7}^{+3.4}-$ & - & $1.14 / 75$ & 0.3 \\
G & $28.4 \pm 2.1$ & $0.2 \pm 0.1$ & $28.4_{-1.9}^{+2.1}$ & - & - & $1.05 / 114$ & 1.3 \\
\hline
\end{tabular}


Table A.4. Same as Table A.1, but for the case of IGR J173543255 observation 0693900201 (see Fig. 9), 0701230101 (see Fig. 11) and 0701230701 (see Fig. 12).

\begin{tabular}{|c|c|c|c|c|c|c|c|}
\hline s. & $N_{\mathrm{H}}^{a}$ & $\Gamma$ & Flux & $C_{\mathrm{MOS} 1}$ & $C_{\mathrm{MOS} 2}$ & $\begin{array}{l}\chi_{\text {red }}^{2} / \\
\text { d.o.f. }\end{array}$ & $\begin{array}{l}\text { Exp. } \\
\text { (ks) }\end{array}$ \\
\hline \multicolumn{8}{|c|}{ Observation 0693900201} \\
\hline $\mathrm{A}^{a}$ & $7.7 \pm 0.2$ & $1.21 \pm 0.05$ & $7.5_{-0.2}^{+0.3}$ & - & - & $0.94 / 673$ & 7.5 \\
\hline $\mathrm{B}^{a}$ & $11.7 \pm 0.7$ & $1.4 \pm 0.1$ & $3.3_{-0.3}^{+0.4}$ & - & - & $1.00 / 217$ & 5.9 \\
\hline$C^{a}$ & $22.6 \pm 2.0$ & $1.2 \pm 0.2$ & $5.1_{-0.8}^{+1.2}$ & - & - & $1.04 / 108$ & 2.8 \\
\hline $\mathrm{D}^{a}$ & $12.2 \pm 1.1$ & $1.1 \pm 0.2$ & $4.1_{-0.5}^{+0.6}$ & - & - & $0.96 / 123$ & 2.6 \\
\hline$E^{a}$ & $8.9 \pm 0.3$ & $1.32 \pm 0.06$ & $7.9 \pm 0.3$ & - & - & $0.98 / 692$ & 7.9 \\
\hline \multicolumn{8}{|c|}{ Observation 0701230101} \\
\hline A & $6.6_{-1.5}^{+1.9}$ & $1.5 \pm 0.4$ & $0.9_{-0.2}^{+0.3}$ & $1.1 \pm 0.2$ & $0.9 \pm 0.2$ & $0.94 / 27$ & 0.9 \\
\hline B & $8.6_{-1.3}^{+1.5}$ & $1.4 \pm 0.2$ & $2.0 \pm 0.3$ & $1.1 \pm 0.1$ & $1.0 \pm 0.1$ & $1.04 / 76$ & 1.2 \\
\hline C & $5.8 \pm 0.5$ & $1.1 \pm 0.1$ & $4.1 \pm 0.2$ & $1.05 \pm 0.07$ & $1.00 \pm 0.06$ & $0.82 / 172$ & 1.6 \\
\hline 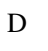 & $5.4_{-1.4}^{+1.7}$ & $1.2 \pm 0.4$ & $0.6 \pm 0.1$ & $1.0 \pm 0.2$ & $1.0 \pm 0.2$ & $0.59 / 22$ & 1.1 \\
\hline$E$ & $7.7_{-1.0}^{+1.1}$ & $1.2 \pm 0.2$ & $1.0 \pm 0.1$ & $1.0 \pm 0.1$ & $1.0 \pm 0.1$ & $0.79 / 97$ & 3.3 \\
\hline 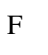 & $6.4_{-0.5}^{+0.6}$ & $1.3 \pm 0.1$ & $3.1 \pm 0.2$ & $1.07 \pm 0.07$ & $1.09 \pm 0.07$ & $1.17 / 188$ & 2.1 \\
\hline G & $7.7_{-2.6}^{+3.0}$ & $1.1 \pm 0.4$ & $1.6_{-0.3}^{+0.5}$ & $1.0 \pm 0.2$ & $1.0 \pm 0.2$ & $0.52 / 28$ & 0.7 \\
\hline$H$ & $5.8_{-1.4}^{+1.8}$ & $1.1 \pm 0.3$ & $2.0_{-0.3}^{+0.4}$ & $1.2 \pm 0.2$ & $1.1 \pm 0.2$ & $1.23 / 26$ & 0.4 \\
\hline C & $9.0_{-1.1}^{+1.3}$ & $1.5 \pm 0.2$ & $1.8_{-0.2}^{+0.3}$ & $1.0 \pm 0.1$ & $1.1 \pm 0.1$ & $0.88 / 107$ & 2.0 \\
\hline 4 & $6.0_{-1.0}^{+1.3}$ & $.0 \pm 0.3$ & $2.3_{-0.2}^{+0.3}$ & $1.0 \pm 0.1$ & $0.9 \pm 0.1$ & $0.70 / 55$ & 0.7 \\
\hline \multicolumn{8}{|c|}{ Observation 0701230701} \\
\hline$x^{2}+2$ & 1. & $1.4 \pm 0.1$ & $2.4 \pm 0.1$ & $1.07 \pm 0.07$ & $1.05 \pm 0.07$ & $0.95 / 175$ & 2.3 \\
\hline B & $6.4 \pm 0.9$ & $1.4 \pm 0.2$ & $1.9 \pm 0.2$ & $1.1 \pm 0.1$ & $1.1 \pm 0.1$ & $1.01 / 56$ & 0.9 \\
\hline $\mathrm{C}$ & $5.0 \pm 0.6$ & $1.2 \pm 0.2$ & $2.0 \pm 0.2$ & $1.2 \pm 0.1$ & $1.1 \pm 0.1$ & $1.26 / 103$ & 1.2 \\
\hline$P_{1}+2$ & $5.0 \pm 0.6$ & $1.4 \pm 0.2$ & $1.2 \pm 0.2$ & $1.2 \pm 0.1$ & $1.1 \pm 0.1$ & $1.09 / 93$ & 1.8 \\
\hline $\mathrm{E}$ & $4.9_{-1.3}^{+1.5}$ & $0.9 \pm 0.3$ & $0.9 \pm 0.1$ & $1.0 \pm 0.2$ & $1.1 \pm 0.2$ & $0.77 / 39$ & 1.7 \\
\hline & $5.4 \pm 0.3$ & $1.3 \pm 0.1$ & $3.8 \pm 0.2$ & $1.00 \pm 0.05$ & $1.00 \pm 0.05$ & $1.06 / 237$ & 2.5 \\
\hline G & $6.1_{-1.4}^{+1.6}$ & $1.1 \pm 0.3$ & $3.7_{-0.4}^{+0.6}$ & $1.0 \pm 0.1$ & $0.9 \pm 0.1$ & $1.01 / 49$ & 0.4 \\
\hline $\mathrm{H}$ & $8.4_{-1.5}^{+1.8}$ & $1.1 \pm 0.3$ & $4.2_{-0.5}^{+0.7}$ & $1.0 \pm 0.1$ & $1.0 \pm 0.1$ & $1.02 / 62$ & 0.6 \\
\hline $\mathrm{L}$ & $5.3 \pm 0.5$ & $0.8 \pm 0.1$ & $5.7 \pm 0.3$ & $1.02 \pm 0.07$ & $1.04 \pm 0.07$ & $1.02 / 187$ & 1.1 \\
\hline M & $5.8 \pm 0.4$ & $1.1 \pm 0.1$ & $5.0 \pm 0.2$ & $1.05 \pm 0.05$ & $1.03 \pm 0.05$ & $1.24 / 236$ & 1.8 \\
\hline N & $6.4_{-0.5}^{+0.6}$ & $1.3 \pm 0.1$ & $2.8 \pm 0.2$ & $1.00 \pm 0.06$ & $1.04 \pm 0.06$ & $0.98 / 186$ & 2.5 \\
\hline D & $5.2 \pm 0.4$ & $1.4 \pm 0.1$ & $4.5 \pm 0.3$ & $1.05 \pm 0.07$ & $1.01 \pm 0.07$ & $1.03 / 167$ & 1.2 \\
\hline
\end{tabular}

Notes. ${ }^{(a)}$ For these spectra no EPIC-pn data were available and the two MOS spectra were merged together to improve the $\mathrm{S} / \mathrm{N}$.
Table A.5. Same as Table A.1, but for the case of IGR J16328-4732 observation 0679810201 (see Fig. 13) and 0679810301 (see Fig. 14).

\begin{tabular}{llllllll}
\hline \hline S. & $N_{\mathrm{H}}$ & $\Gamma$ & Flux & $C_{\mathrm{MOS} 1}$ & $C_{\mathrm{MOS} 2}$ & $\begin{array}{c}\chi_{\text {red }}^{2} / \\
\text { d.o.f. }\end{array}$ & $\begin{array}{c}\text { Exp. } \\
(\mathrm{ks})\end{array}$ \\
\hline &
\end{tabular}

\begin{tabular}{|c|c|c|c|c|c|c|}
\hline & & $\pm 0.1 \quad 15.6_{-0.8}^{+0.9}$ & 3 & 4 & 79 & \\
\hline & & \pm 0.52 . & & & & 11 \\
\hline & & \pm 0.23 & & .06 & 58 & $5.1^{7}$ \\
\hline & \pm 1.7 & 0.23 & & $1.06 \pm$ & $1.02 / 13$ & 2.5 \\
\hline & & $\pm 0.5 \quad 7.0_{-1.7}^{+3.4}$ & & 2 & & .1 \\
\hline & & $.29 .2_{-1.4}^{+2.0}$ & 99 & $1.09 \pm$ & $0.80 / 106$ & 0.68 \\
\hline & & $.412 .7_{-3.1}^{+5.3}$ & & 2 & 51 & 0.2 \\
\hline & & ${ }_{-0.4}^{+0.6}$ & 7 & 7 & 45 & 1.92 \\
\hline & & 0.4 & & , & & 2.18 \\
\hline & & $\pm 0.45 .8_{-1.1}^{+1.8}$ & & & & 0.3 \\
\hline & & $\pm 0.18 .4 \pm 0.5$ & 0 & 05 & & 1.60 \\
\hline & 5 & $2 \pm 0.4 \quad 4.9_{-1.1}^{+1.8}$ & $0+8+8-1$ & 0 & & 0.4 \\
\hline & & $1.5 \pm 0.4 \quad 1.1_{-0.2}^{+0.4}$ & $1.1 \pm 0.1$ & $0+0+0+2$ & $.97 / 47$ & 2.2 \\
\hline & & $1.5 \pm 0.5 \quad 1.3_{-0.3}^{+0.6}$ & $1.1 \pm 0.2$ & 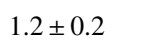 & 34 & 1.44 \\
\hline & & $1.8 \pm 0.42 .2_{-0.6}^{+1.1}$ & $0.9 \pm 0$ & 0 & & 1.39 \\
\hline & & $0.2 \quad 1.9 \pm 0.2$ & & & & 5.49 \\
\hline \multicolumn{7}{|c|}{ Observation 0679810301} \\
\hline & & .7 & & & & 1.04 \\
\hline & & $1.6 \pm 0.5 \quad 0.5_{-0.1}^{+0.3}$ & & & & 3.27 \\
\hline & & $1.7 \pm 0.5 \quad 2.2_{-0.6}^{+1.2}$ & & & & 1.18 \\
\hline & & $0.3_{-0.4}^{+0.6} \quad 1.4_{-0.2}^{+0.3}$ & & & & 0.46 \\
\hline & & $1.1 \pm 0.2 \quad 2.3_{-0.2}^{+0.3}$ & & & & 2 \\
\hline & & $0.8 \pm 0.42 .2_{-0.3}^{+0.5}$ & & & & 0.78 \\
\hline & & $1.2 \pm 0.4 \quad 4.8_{-0.9}^{+1.3}$ & & & & 0.50 \\
\hline & & $1.6 \pm 0.4 \quad 14.6_{-3.0}^{+5.4}$ & & & & 0.2 \\
\hline & & $0.9 \pm 0.24 .8_{-0.6}^{+0.8}$ & & & & 0.93 \\
\hline & & $1.1 \pm 0.4 \quad 1.2_{-0.2}^{+0.4}$ & & & & 2.22 \\
\hline & & $1.3 \pm 0.1 \quad 12.3_{-0.9}^{+1.0}$ & 0.87 & & & 1 \\
\hline & & $1.6 \pm 0.46 .8_{-1.4}^{+2.5}$ & & & & 0.41 \\
\hline & & $1.5 \pm 0.23 .3_{-0.4}^{+0.5}$ & $1.00 \pm$ & $1.00 \pm 0.08$ & 0.9 & 2.24 \\
\hline & & $1.0_{-0.5}^{+0.3}$ & 1. & 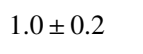 & $1.15 / 30$ & 1.64 \\
\hline & $13.1 \pm 0.8$ & $1.2 \pm 0.1 \quad 4.0 \pm 0.3$ & $0.30 \pm 0.0 J$ & $1.08 \pm 0.05$ & & (1) \\
\hline
\end{tabular}

